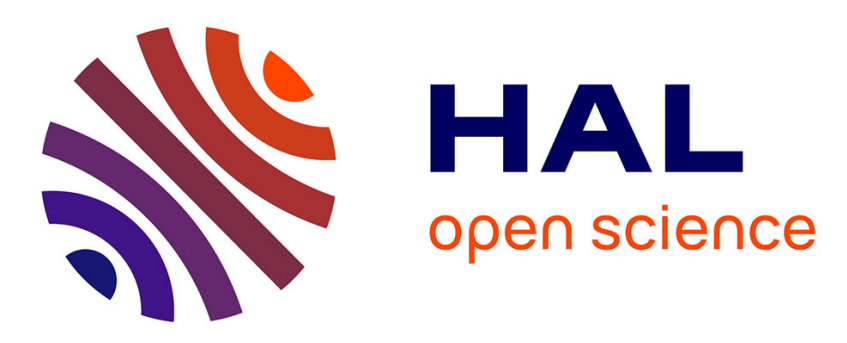

\title{
Investigating the hydromechanical behaviour of bentonite pellets by swelling pressure tests and discrete element modelling
}

Benjamin Darde, Jean-Noël Roux, Jean-Michel Pereira, Patrick Dangla, Jean Talandier, Minh Ngoc Vu, Anh Minh A.M. Tang

\section{To cite this version:}

Benjamin Darde, Jean-Noël Roux, Jean-Michel Pereira, Patrick Dangla, Jean Talandier, et al.. Investigating the hydromechanical behaviour of bentonite pellets by swelling pressure tests and discrete element modelling. Acta Geotechnica, 2021, 16 (2), pp.507-524. 10.1007/s11440-020-01040-5 . hal03053589

\section{HAL Id: hal-03053589 \\ https://hal-enpc.archives-ouvertes.fr/hal-03053589}

Submitted on 11 Dec 2020

HAL is a multi-disciplinary open access archive for the deposit and dissemination of scientific research documents, whether they are published or not. The documents may come from teaching and research institutions in France or abroad, or from public or private research centers.
L'archive ouverte pluridisciplinaire HAL, est destinée au dépôt et à la diffusion de documents scientifiques de niveau recherche, publiés ou non, émanant des établissements d'enseignement et de recherche français ou étrangers, des laboratoires publics ou privés. 


\section{TITLE:}

Investigating the hydromechanical behaviour of bentonite pellets by swelling pressure tests and discrete element modelling.

\section{AUTHORS:}

1) DARDE Benjamin

Affiliation : (1) Navier, Ecole des Ponts, Univ Gustave Eiffel, CNRS, Marne-la-Vallée, France; and (2) French National Radioactive Waste Management Agency (Andra), Châtenay-Malabry, France

Address: Ecole des Ponts ParisTech, Laboratoire Navier, 6-8 avenue Blaise Pascal, 77455

Marne-la-Vallée, France

Email: benjamin.darde@enpc.fr

2) ROUX Jean-Noël

Affiliation: Navier, Ecole des Ponts, Univ Gustave Eiffel, CNRS, Marne-la-Vallée, France

Address: Ecole des Ponts ParisTech, Laboratoire Navier, 2 allée Kepler, 77420 Champs-sur-

Marne, France

Email: jean-noel.roux@ifsttar.fr

3) PEREIRA Jean-Michel

Affiliation: Navier, Ecole des Ponts, Univ Gustave Eiffel, CNRS, Marne-la-Vallée, France

Address: Ecole des Ponts ParisTech, Laboratoire Navier, 6-8 avenue Blaise Pascal, 77455

Marne-la-Vallée, France

Email: jean-michel.pereira@enpc.fr

4) DANGLA Patrick

Affiliation: Navier, Ecole des Ponts, Univ Gustave Eiffel, CNRS, Marne-la-Vallée, France Address: Ecole des Ponts ParisTech, Laboratoire Navier, 6-8 avenue Blaise Pascal, 77455

Marne-la-Vallée, France

Email: patrick.dangla @ifsttar.fr

5) TALANDIER Jean

Affiliation: French National Radioactive Waste Management Agency (Andra), ChâtenayMalabry, France

Address: Andra, DRD/MFS, 1-7 rue Jean Monnet, Parc de la croix blanche, 92298 ChâtenayMalabry, France

Email: jean.talandier@andra.fr

6) VU Minh Ngoc

Affiliation: French National Radioactive Waste Management Agency (Andra), Châtenay-

Malabry, France

Address: Andra, DRD/MFS, 1-7 rue Jean Monnet, Parc de la croix blanche, 92298 Châtenay-

Malabry, France

Email: minh-ngoc.vu@andra.fr

7) TANG Anh Minh*

Affiliation: Navier, Ecole des Ponts, Univ Gustave Eiffel, CNRS, Marne-la-Vallée, France

Address: Ecole des Ponts ParisTech, Laboratoire Navier, 6-8 avenue Blaise Pascal, 77455

Marne-la-Vallée, France

Email: anh-minh.tang@enpc.fr

${ }^{*}$ Corresponding author.

\section{ACKNOWLEDGEMENTS}


The present work is supported by the French National Radioactive Waste Management Agency (Andra), which is gratefully acknowledged.

\section{ABSTRACT}

Bentonite pellet mixtures are candidate material for the sealing of galleries in radioactive waste disposals. The hydromechanical behaviour of assemblies of bentonite pellets is investigated upon partial hydration through (I) suction-controlled swelling pressure tests in the laboratory and (ii) discrete element method (DEM) simulations. The combination of these experimental and numerical approaches highlights that, before the mixture homogenisation, the swelling pressure develops in two phases. The first phase is characterised by an increase of contact forces between pellets. The second phase is characterised by plasticity at contacts between pellets and is controlled by the progressive decrease of pellet strength and stiffness upon hydration. In addition, numerical results highlight that the swelling pressure measured in the laboratory can be influenced by the sample preparation, the cell size, and the diameter of the pressure sensor.

Keywords: Expansive soils; Pellets; Swelling pressure; DEM simulations 


\section{1. Introduction}

3 Compacted bentonite-based materials are a candidate material for engineered barriers in radioactive waste disposal concepts due to their low permeability, good radionuclide retention capacity and ability to swell upon hydration, resulting in the filling of technological voids and a swelling pressure development on the tunnel walls. The development of a swelling pressure contributes to the decrease of hydraulic conductivity in the excavation induced damaged zone. In this respect, the hydromechanical behaviour of compacted bentonite materials has been widely studied over the last two decades [1-16].

The use of pellet-based bentonite materials has been considered an interesting solution for the installation of engineered barriers because of operational convenience [17-27]. Pellet materials are laid as a dry granular assembly in the galleries and subsequently undergo swelling upon hydration from the host rock pore water. Upon hydration, pellet materials are known to lose their initial granular structure and reach a homogeneous final state $[18,19,27]$. Like other bentonite materials, the final swelling pressure developed by pellet materials in constant volume conditions can be estimated from their initial dry density [28, 29].

Before homogenisation, the hydromechanical behaviour of pellet materials is still not perfectly understood. Upon pellet swelling, the swelling pressure is influenced by the granular nature of the material $[28,29]$. Interactions at contacts between pellets and stiffness decrease of the pellets during hydration are thought to control the overall hydromechanical behaviour [31].

It is proposed in the present study to evaluate the influence of interactions at contacts on the macroscopic response of bentonite pellet assemblies upon hydration. The objective is to obtain an insight into the hydromechanical behaviour of pellet materials upon hydration, before reaching a homogeneous state. In this purpose, swelling pressure tests are performed in the laboratory on pellet materials. Discrete Element Method (DEM) is then used to model the swelling pressure tests. 
31 In DEM simulations [32], each grain is modelled individually. DEM allows to study the influence of the behaviour of one pellet and interactions at contacts between pellets on the behaviour of the granular assembly. DEM has been successfully used in similar engineering problems involving irreversible changes in the volume of grains, such as the effect of particle thermal expansion in granular materials [33,34]; or grain-scale modelling of the swelling behaviour of absorbent polymer particles upon hydration in hygienic products [35].

The ability of DEM to access grain-scale phenomena can provide valuable information about the behaviour of assemblies of pellets upon hydration-induced pellet swelling. These latter can be obtained neither through experimental tests, since only macroscopic phenomena are measurable most of the time, nor through finite element method (FEM) simulations, which require a homogeneous continuum. In the present study, DEM is an interesting tool to study the hydromechanical behaviour of pellet materials before homogenisation while providing a better understanding of the results of laboratory tests.

The present paper applies DEM to the simulation of the partial hydration of expansive clay pellet assemblies. In DEM, the behaviour of each pellet is based on the hydromechanical model for a single pellet proposed by Darde et al. (2018) [36]. Interactions between pellets are described by contact mechanics and characterised by the Hertz law and the Coulomb friction [37]. Some preliminary results obtained using this approach can be found in Darde et al. (2020) [38]. In the present work, more comprehensive description, analysis and discussion of the experimental study and numerical simulations are presented. The modelling approach is validated by simulating suction-controlled swelling pressure tests carried out at laboratory scale.

Materials and experimental methods regarding the swelling pressure tests are first described. The main features of the DEM simulations are presented in the second part. Both experimental and numerical results are then presented and discussed in the third and fourth parts, respectively. Important contributions and main perspectives arising from the present study then constitute the paper conclusion.

60 


\section{Material and Experimental methods}

62

\subsection{Bentonite pellets}

64

The material studied in the framework of the present study is an assembly of MX80 bentonite granules or pellets. MX80 is a sodium (Na)-bentonite from Wyoming (USA). Its main properties are summarised in Table 1.

68

Pellets are obtained from MX80 bentonite powder by pressure casting (compaction in a mould). Granules are composed of a central cylinder with spherical caps at both ends. Their initial dry density, suction value and water content are $1.90 \mathrm{~g} / \mathrm{cm}^{3}, 89 \mathrm{MPa}$ and 0.122 , respectively [36]. The main initial properties of the pellets are listed in Figure 1 and Table 2.

\subsection{Constant-volume swelling pressure tests}

\subsubsection{Pellet assembly}

In the French concept of radioactive waste disposal, a bentonite pellet and powder (crushed pellets) mixture is candidate material for the sealing plugs preventing fluid migrations. The dry density of the reference mixture and the dry mass proportion of pellets and powder are 1.50 $\mathrm{g} / \mathrm{cm}^{3}$ and $70 / 30$, respectively. The mixture is laid in a dry state as a granular assembly. Interactions between high-density granules are thought to have an influence on the material mechanical behaviour at the beginning of hydration, especially at the structure scale if heterogeneity of powder content arises [22], as a consequence of either an imperfect laying or particle segregation. response at the beginning of hydration is studied through constant-volume swelling pressure tests carried out on a pellet assembly without powder. The material dry density in the present study is thus $1.05 \mathrm{~g} / \mathrm{cm}^{3}$. The initial pellet volume fraction is 0.553 . 
94 The swelling pressure in the present study is defined as the pressure developed by the material against the upper wall of a cylindrical isochoric cell upon hydration. A pressure sensor (BER-A5MP15S from Kyowa, capacity of $5 \mathrm{MPa}$ ) at the centre of the cell upper wall measures the swelling pressure as a function of elapsed time.

The cylinder dimensions are given in Table 3. A sketch of the cell is presented in Figure 2.

2.2.3. Sample preparation

101

102 Two samples are prepared (referred to as SP1 and SP2 in the following). In both cases, the

103 target dry density $\left(1.05 \mathrm{~g} / \mathrm{cm}^{3}\right)$ is reached by introducing a mass of pellets of $99.9 \mathrm{~g}$. This mass corresponds to 209 pellets. The 209 pellets are placed one by one in the cell in successive layers [22]. Cells are then closed by placing the upper wall so that the cell height is $30 \mathrm{~mm}$. The closure step can induce a pressure increase if upper wall-pellet contacts are created. The preparation step of SP1 and SP2 resulted in an initial pressure of $10 \mathrm{kPa}$ and $55 \mathrm{kPa}$ after the cell closure, respectively.

2.2.4. Suction-controlled hydration at constant room temperature

112 Hydration is performed step by step through the vapour equilibrium technique as described in

$113[2,19,39]$. Suction is decreased from $89 \mathrm{MPa}$ (initial state) to $4 \mathrm{MPa}$ (SP1 test) and $9 \mathrm{MPa}$

114 (SP2 test). Salt solutions used in the study are listed in Table 4 along with their corresponding 115 suction values.

117 A peristaltic pump makes air circulate through the salt solution to impose a constant target

118 relative humidity, then through the isochoric cell. Humid air is allowed to circulate directly from 119 the bottom to the top of the cell through a side tube. Thus, no excessive air pressure is 
developed when using the peristaltic pump and humid air is considered to circulate freely inside the inter-pellet porosity (Figure 3). Room temperature is $20^{\circ} \mathrm{C} \pm 1^{\circ} \mathrm{C}$.

123 As humid air circulates in the isochoric cell, pellets are hydrated and swell. Pellet swelling in an

124 isochoric cell results in the development of a swelling pressure which is measured by the pressure sensor at the centre of the top wall.

127 Equilibrium is considered to be reached when the pressure measured by the sensor remains

128 constant for a minimum of 3 days. The final pressure value is used to draw the suction-swelling pressure relationship. The current salt solution is then replaced by the following one (Table 4) to

130 impose the next relative humidity step.

\section{Discrete Element Method simulations of the swelling pressure tests}

\subsection{Overview of the method}

DEM simulations are carried out by using an in-house program [40]. In the DEM simulations carried out, each pellet is modelled as a sphere. A molecular dynamics scheme in the quasistatic limit is used [40]. The hydromechanical behaviour of each pellet upon hydration is described by the model proposed by [36] for a single pellet.

The sphere diameter $a_{e q}$ is chosen such that its volume coincides with the pellet volume $V$ :

$$
a_{e q}=\left(\frac{6 V}{\pi}\right)^{1 / 3}
$$

145 The initial value of $a_{e q}$ is obtained from initial values of mass and density: $a_{e q} 0=7.53 \mathrm{~mm}$.

147 In order to assess the ability of the model to reproduce the hydromechanical behaviour of the

148 granular assembly, swelling pressure tests are simulated. In the simulations, the isochoric cell is 
modelled as a rigid cylinder (infinite Young's modulus), the height and diameter of which are the same as the cell used for the swelling pressure tests. 209 pellets are simulated in each

151 simulations, corresponding to the number of granules in the swelling pressure tests performed

152 in the laboratory, thus the same pellet volume fraction.

Simulations are carried out in two main steps: cylinder and the cell closure;

Three different types of simulations are carried out and referred to as DEM1; DEM2; DEM3. For each type, 100 calculations are performed to quantify the variability of the results. The difference between the simulations is the initial position of the beads following the sample preparation. This point is described in a dedicated section. DEM1 simulations aim at reproducing the swelling pressure tests with optimised preparation to avoid initial pressure (no friction during preparation). DEM2 simulations aim at assessing the influence of a less optimised initial state following the preparation step on the material behaviour upon hydration (friction during preparation). In both DEM1 and DEM2, contact friction is taken into account during hydration. In DEM3 simulations, no contact friction is taken into account during both preparation and hydration steps (Table 5). The following parts provide details concerning the model for a pellet, contact laws, hydration and preparation steps.

\subsection{Hydromechanical behaviour of a pellet}

175 From grain-level experimental characterisation, Darde et al. (2018) [36] proposed a model 176 describing the evolution of pellet stiffness, volumetric strain and strength upon suction decrease

177 (Figures $4 \mathrm{a}$ and $4 \mathrm{~b}$ ). Pellets are characterised by their microstructure, assumed to be fully178 saturated. The following notations are used for the model parameters: 
$180 \quad-\quad v$ is the pellet Poisson ratio, taken equal to 0.3 ;

$181-\varepsilon_{V m}$ is the volumetric strain;

$182-s_{0}$ is the initial pellet suction;

$183-R_{A}$ is the strength;

$184-\alpha_{m} ; \beta_{m}$ and $C_{A}$ are model parameters, respectively taken equal to $0.024 \mathrm{MPa}^{-1} ; 0.016$ $\mathrm{MPa}^{-1}$ and $0.12 \mathrm{~N} / \mathrm{MPa}[36]$

$186 \quad-\quad p^{\prime}$ is the effective mean stress. Since pellets are considered fully-saturated, $p^{\prime}$ is taken equal to the sum of the total mean stress $p$ and the suction $s$. In the considered suction range, $s \gg p$ and it is assumed that $p^{\prime}=s$.

189 Using these notations, the model equations describing the pellet behaviour are:

190

$$
\begin{gathered}
E_{m}\left(p^{\prime}\right)=3(1-2 v) \frac{1}{\beta_{m}} \exp \left(\alpha_{m} p^{\prime}\right) \\
\varepsilon_{V m}\left(p^{\prime}\right)=\frac{\beta_{m}}{\alpha_{m}}\left[\exp \left(-\alpha_{m} s_{0}\right)-\exp \left(-\alpha_{m} p^{\prime}\right)\right]
\end{gathered}
$$

(4)

$$
R_{A}\left(p^{\prime}\right)=C_{A} E_{m}\left(p^{\prime}\right)
$$

$$
p^{\prime}=p+s=s
$$

191

192 The above equations are used in DEM to determine the mechanical properties of each particle

193 at any value of suction.

\subsection{Hydration modelling}

197

198 Pellet hydration and subsequent swelling are simulated by a simultaneous increase of all the

199 bead diameters. It is therefore assumed that all pellets get hydrated at the same rate. The

200 diameter increase at each hydration step $i$ varies from $0.1 \%$ to $0.9 \%$ from initial state $(89 \mathrm{MPa}$ 
201 of suction) to final state (9 MPa of suction). 60 diameter increments are imposed, for an overall 202 diameter increase of $\sim 15 \%$ :

203

(6)

$$
a_{e q \mathrm{i}+1}=\lambda_{i} a_{e q \mathrm{i}}
$$

204

205 Where:

206

$$
\lambda_{i}=\exp \left[\ln \left(\frac{\lambda_{f}}{\lambda_{0}}\right)\left(\frac{a_{e q i}-a_{e q 0}}{a_{e q f}-a_{e q 0}}\right)+\ln \left(\lambda_{0}\right)\right]
$$

207

208 Where $\lambda_{i}$ characterises the diameter increment; $\lambda_{f}$ and $\lambda_{0}$ are parameters taken equal to $10^{-2}$ and

$20910^{-3}$, respectively; $a_{e q} i$ and $a_{\text {eq }}$ are the equivalent diameters at step $i$ and at the final suction

210 value, respectively.

211

212 From radius increments, the volume variation is obtained. The suction value corresponding to

213 each hydration stage is obtained from equation (3). Stiffness and strength are obtained from

214 equations (2) and (4), respectively.

215

216 Contact laws then allow the granular assembly evolution to be computed, taking into account

217 the suction-dependency of the aforementioned material parameters.

218

219

\subsection{Contact laws}

220

221

In contacts between two granules of same radius and modulus, the Hertz law (Figure 5) relates

222 the normal contact elastic force $F_{N}$ to the normal elastic deflection at contact $\delta_{N}{ }^{e}$ as:

223

224

(8)

$$
F_{N}=\frac{1}{3} \frac{E_{m}}{1-v^{2}} a_{e q}^{1 / 2} \delta_{N}^{e 3 / 2}
$$

225 Contacts between a granule and the infinitely stiff flat walls satisfy: 
(9)

$$
F_{N}=\frac{2^{3 / 2}}{3} \frac{E_{m}}{1-v^{2}} a_{e q}^{1 / 2} \delta_{N}^{e}{ }^{3 / 2}
$$

228 Granule strength is taken into account by considering perfect plasticity at contacts. The elastic

229 part is described by the Hertz law (equations (8) and (9)) and the elastic limit is given by

230 equation (4). Thus,

231

(10)

$$
F_{N} \leq R_{A}
$$

233 and plastic deflection can arise for $F_{N}=R_{A}$ (Figure 6a).

234

235 Tangential elasticity at contacts is described by a simplified form of the Cattaneo-Mindlin-

236 Deresiewicz laws [37], with due care for energetic consistency and objectivity, as proposed by

237 Agnolin and Roux (2007) [40]. $\dot{F}_{\boldsymbol{T}}$, the increment of tangential reaction vector $\boldsymbol{F}_{\boldsymbol{T}}$, is related to

$238 \dot{\boldsymbol{\delta}}_{\boldsymbol{T}}$, the increment of relative tangential displacement vector $\boldsymbol{\delta}_{\boldsymbol{T}}$, using the following equation:

239

240

$$
\dot{\boldsymbol{F}}_{T}=\frac{2-2 v}{2-v} \frac{\mathrm{d} F_{N}}{\mathrm{~d} \delta_{N}} \dot{\boldsymbol{\delta}_{T}}
$$

241 The Coulomb condition is checked so that

$$
F_{T} \leq \mu F_{N}
$$

243

244 where $F_{T}$ is the norm of the $\boldsymbol{F}_{T}$ vector and $\mu$ is a friction coefficient.

245 Sliding can arise for $F_{T}=\mu F_{N}$ (Figure 6b). The same value of $\mu$ is taken for both pellet-pellet

246 and pellet-wall contacts.

248 Damping is taken into account by adding a viscous component to the hertzian elastic force. The

249 viscous force, $N^{v}$, in a contact between two pellets is written as: 


$$
N^{v}=\alpha_{v}\left(2 m_{p} \frac{\mathrm{d} F_{N}}{\mathrm{~d} \delta_{N}}\right)^{1 / 2} \frac{\mathrm{d} \delta_{N}}{\mathrm{~d} t}
$$

252 Where $\alpha_{v}$ is a fraction of the critical damping coefficient and $m_{p}$ is the mass of the pellet. $\alpha_{v}$ is taken equal to 0.9. Further discussion concerning the damping in contacts can be found in [40].

\subsection{Numerical sample preparation}

Numerical samples are prepared by placing pellets one by one in a rigid cylinder. The initial height of the cylinder, $H_{c e l l}$, is set at a value of $H_{c e l l}+a_{e q} 0$, where $H_{c e l l}$ is the cylinder height during the swelling step. This initial value ensures that all the pellets can be placed in the cylinder. A pellet is placed in the cell each second. The first 20 pellets are randomly placed at the bottom of the cell. Then, each bead is placed at a position corresponding to the lowest elevation in the cell to reproduce experimental preparation (Figure 7a). During this process, the granular assembly is constantly being equilibrated under gravity. When all the pellets are placed

264 in the cell, calculation continues until equilibrium of forces and moments is reached. The cell closure is then simulated by incrementally decreasing the cylinder height from $H_{\text {cell } 0}$ to $H_{c e l l}$.

266 Each height decrease is followed by the calculation of a new equilibrium under gravity before a new decrease. The initial solid fraction of all the numerical samples is the same as in experiment $(0.553$, corresponding to an inter-pellet porosity of 0.447$)$.

\subsection{Equilibrium of forces and moments}

272 At each calculation step, i.e. height decrease during preparation or new suction value during

273 hydration, the granular assembly is considered at equilibrium if both the ratio of the net force

$274 F_{\text {net }}$ to the maximum normal force $F_{\max }$ and the ratio of the net moment $\Gamma_{\text {net }}$ to the maximum 275 moment $\Gamma_{\max }$ ratio are, on each bead, below a tolerance value $\epsilon$ :

$$
\left|F_{\text {net }}\right| \leq \epsilon\left|F_{\text {max }}\right|
$$




$$
\left|\Gamma_{\text {net }}\right| \leq \epsilon\left|F_{\text {max }}\right| \frac{a_{e q}}{2}
$$

278

279

$\epsilon$ is taken equal to $10^{-4}$ in all the simulations.

280

281

3.7. Swelling pressure calculation

282

283 At equilibrium, the swelling pressure $P_{S}^{*}$ is computed as the total normal force exerted by the material onto the sensor, divided by the sensor area (Figure 7b). The sensor area is at the centre of the upper wall and defined by the sensor diameter $D_{\text {sensor }}$. Different values of swelling pressure can be calculated from one simulation, depending on the value of $D_{\text {sensor }}$.

\section{Results}

289

\subsection{Experimental suction-swelling pressure relationship}

292 The evolution of the swelling pressure is measured as a function of elapsed time. Following a

293 change of imposed suction value, the pressure increases or decreases, then reaches a plateau.

294 For each suction value, the plateau value is retained as the corresponding swelling pressure. A

295 new suction value is then imposed (Figure 8a and 8b). Some pressure fluctuations are

296 measured, and are thought to be related to experimental conditions. Room temperature and

297 humidity variations, slight voltage variations in the measurement apparatus, or opening of the

298 bottle containing salt solutions for cleaning (thus temporary modifying the imposed suction), are

299 examples of external perturbations which influence the measurements. Regular cleaning of the

300 bottle was actually compulsory as salt crystallisation on the bottle side would increase the

301 suction, and salt crystallisation inside the tube plunged in the solution would prevent air

302 circulation. 
304 Following the cell closure, SP1 initial pressure was $10 \mathrm{kPa}$ at initial suction $89 \mathrm{MPa}$. Equilibrium 305 then required $\sim 30 ; \sim 120 ; \sim 75 ; \sim 80 ; \sim 35$ and $\sim 55$ days on imposing $82 ; 59 ; 38 ; 25 ; 9$ and $4 \mathrm{MPa}$, 306 respectively. Following the cell closure, SP2 initial pressure was $55 \mathrm{kPa}$. Equilibrium in this test required less time, as an overall plateau value was reached at higher suction, resulting in little pressure variations on imposing new suction values. On imposing 82; 59; 40;38; 25; 13 and 9 $\mathrm{MPa}$, equilibrium required $\sim 30 ; \sim 10 ; \sim 20 ; \sim 20 ; \sim 15 ; \sim 25$ and $\sim 30$ days to be reached, respectively.

312 The suction-swelling pressure relationships obtained for SP1 and SP2 samples are presented in

313 Figure 9. Results highlighted that upon hydration under constant-volume conditions, the

314 swelling pressure of pellet materials increased in two phases within the considered suction

315 range. The first phase is characterised by an increase of swelling pressure. The second phase

316 is characterised by either a plateau or a decrease of swelling pressure. In SP1 test, the axial

317 pressure $\left(P_{S \times P}\right)$ increases from $10 \mathrm{kPa}$ (closure pressure) to $130 \mathrm{kPa}$ when suction is

318 decreased from $89 \mathrm{MPa}$ (initial state) to $25 \mathrm{MPa}$. $P_{S \times P}$ slightly increases from $130 \mathrm{kPa}$ to 150

$319 \mathrm{kPa}$ when suction is decreased from $25 \mathrm{MPa}$ to $9 \mathrm{MPa}$, then slightly decreases to $137 \mathrm{kPa}$

320 when suction is decreased to $4 \mathrm{MPa}$. In SP2 test, $P_{S X P}$ increases from $55 \mathrm{kPa}$ (closure

321 pressure) to $180 \mathrm{kPa}$ when suction is decreased from $89 \mathrm{MPa}$ to $25 \mathrm{MPa}$. After this phase, $P_{S \times P}$

322 decreases from 180 to $130 \mathrm{kPa}$ when suction is decreased from $25 \mathrm{MPa}$ to $9 \mathrm{MPa}$.

\section{4.2. Dismantling of experimental samples}

SP1 and SP2 isochoric cells were opened after reaching equilibrium at $4 \mathrm{MPa}$ and $9 \mathrm{MPa}$ of suction, respectively. Both SP1 and SP2 samples appeared to be granular. Pellets and macropores could easily be identified (Figure 10a and 10b). Some pellets in contact with the upper wall were deformed in both samples. It was not possible to determine whether it occurred during the closure or during swelling. 
334 Figure 11 presents the results of swelling pressure as a function of suction obtained in simulations DEM1; DEM2 and DEM3. As a reminder, 100 simulations were carried out for

336 DEM1, DEM2, and DEM3. Only the mean values of swelling pressure are plotted, for a ratio of sensor diameter to initial pellet diameter of 4 (as in experimental tests).

In DEM1, the mean value of swelling pressure increases from 0 to $330 \mathrm{kPa}$ as suction decreases from $89 \mathrm{MPa}$ to $60 \mathrm{MPa}$, then slowly decreases to reach $110 \mathrm{kPa}$ at $4 \mathrm{MPa}$ of suction. In DEM2, swelling pressure reaches $500 \mathrm{kPa}$ during the closure step, then slowly decreases to reach $110 \mathrm{kPa}$ at $4 \mathrm{MPa}$ of suction. In DEM3, the pressure slowly increases to 10 $\mathrm{kPa}$ from $89 \mathrm{MPa}$ to $80 \mathrm{MPa}$ of suction, then increases to $250 \mathrm{kPa}$ at $60 \mathrm{MPa}$ of suction, then slowly decreases to $110 \mathrm{kPa}$ as suction decreases to $4 \mathrm{MPa}$.

\subsection{Grain-scale features of numerical samples}

\subsubsection{Contact plasticity}

The evolution of the number of plastic contacts upon hydration is determined in numerical samples. Figures $11 \mathrm{a}, 11 \mathrm{~b}$ and $11 \mathrm{c}$ present the evolution of the mean plastic contacts proportion for the whole sample, $x_{c t o t}^{p}$, and for contacts between pellets and the upper wall, where pressure is measured, $x_{c \text { sup }}^{p}$, for simulations DEM1, DEM2 and DEM3. Results are presented along with the swelling pressure development upon suction decrease. In all samples, the increase in $x_{c \text { sup }}^{p}$ is more significant than the increase in $x_{c \text { tot }}^{p}$.

In DEM1, from initial state to $70 \mathrm{MPa}$ of suction, the swelling pressure increases while no

358 contact has reached the elastic limit. The number of plastic contacts increases sharply between

$35970 \mathrm{MPa}$ and $60 \mathrm{MPa}$ of suction. This suction range corresponds to the peak swelling pressure.

360 The swelling pressure then slowly decreases, as $x_{c \text { sup }}^{p}$ reaches a maximum and keeps slowly

361 increasing in the whole sample. 
363 The evolution of plastic contacts in DEM3 exhibited a similar trend as in DEM1, but the increase

364 of $x_{c \text { tot }}^{p}$ and $x_{c \text { sup }}^{p}$ was not as sharp as in DEM1. In DEM2, the pressure increase due to the cell

365 closure induced contact plasticity. The influence of the closure step in this case is significant. 25

366 "pellet-upper wall" contacts were created during this step. 15 of these contacts reached the

367 elastic limit during the process, which represents $2.5 \%$ of the overall 600 contacts in the

368 samples. Yet, no significant increase of swelling pressure is recorded upon hydration. The

369 measured pressure keeps decreasing as $x_{c t o t}^{p}$ and $x_{c \text { sup }}^{p}$ increase.

370

$371 \quad$ 4.4.2. Inter-pellet porosity

372

373 At initial state, the porosity was $\sim 0.447$. Upon pellet swelling, porosity in DEM progressively

374 decreases and reaches a value slightly below 0.2 (Figure 12). This latter is below the minimal

375 value for a dense packing of spheres of same diameter. It highlights that total deflection at

376 contact is non-negligible at low suction. Considering plasticity in contacts allows the elastic

377 deflection, used in equations (8) and (9), to remain small and avoids reaching unrealistically

378 high contact forces.

379

380 4.4.3. Coordination number

381

382 The coordination number $Z$, defined by the average number of force-carrying contact per grain,

383 relates to the number of particles $N_{p}$, the number of particle-particle contacts $N_{c}$ and the

384 number of particle-wall contacts $N_{c 2}$ as:

385

386

$$
Z=\frac{2 N_{c 1}+N_{c 2}}{N_{p}}
$$

$387 Z$ is calculated following preparation and upon hydration. Results are presented in Figure 12 for DEM1, DEM2 and DEM3 along with the evolution of inter-pellet porosity. 
390 After closure, the mean coordination number in samples prepared under zero-friction conditions

391 (DEM1 and DEM3) is 5.4. The mean number of contacts in this case is 635 , slightly higher than

$3923 N_{p}$ [41], as it should be for frictionless beads approaching the limit of small deflections.

394 The presence of friction in the assembling stage reduces the number of contacts [40]. Thus samples DEM2 contain 600 contacts on average, with a coordination number of 5.1 .

Upon hydration, the coordination number of frictionless samples (DEM3) increases to a final value of 8.2 (net increase: +2.8 ). The coordination number of both DEM1 and DEM2 samples increased at the same rate, reaching final values of 7.5 and 7.2 respectively (net increase: + 2.1).

401

The coefficient of variation (standard deviation to mean value ratio) of swelling pressure, proportion of plastic contacts and coordination number for the three simulation types is calculated from 100 simulations and presented in Figure 13.

407

The result variability for swelling pressure and proportion of plastic contacts decreased upon hydration for the three types of simulation. At $9 \mathrm{MPa}$ of suction, its value for swelling pressure reached $\sim$ 0.10-0.15 (for $D_{\text {sensor }} / a_{e q}=4$ ). Its value for proportion of plastic contacts reached $\sim 0.03-0.04$. The coefficient of variation of coordination number remained low $(<0.02)$ for the three types of simulation upon hydration.

\subsection{Influence of the sensor diameter}

416 In DEM1, the calculation of swelling pressure from contact forces between pellets and the upper

417 wall is carried out for different values of $D_{\text {sensor }}$. Different suction-swelling pressure relationships

418 are thus obtained. Figure 14 presents the variability of the measured swelling pressure in the

419100 DEM1 simulations performed, for two values of the $D_{\text {sensor }} / a_{e q}$ ratio. In both cases, an 
interval from $m-2 \sigma$ to $m+2 \sigma$ is plotted, with $m$ and $\sigma$ the mean value and the standard

421 deviation of the swelling pressure, respectively. The mean values are very close for both sensor

422 diameters and are not plotted in Figure 14. The standard deviation significantly increases with

423 decreasing sensor diameter.

424

425 The coefficient of variation is determined for six sensor diameter to pellet diameter ratios, at

426 peak measured pressure and at $9 \mathrm{MPa}$ of suction. Results are plotted in Figure 15. The

427 coefficient of variation of the measured swelling pressure reaches 0.8 to 1.3 in the case where

428 both the sensor diameter and the initial pellet diameter are identical.

430

5. Discussion

432

433 The hydromechanical behaviour of expansive clay pellet materials has been studied through

434 swelling pressure tests at laboratory scale and DEM, providing access to grain-scale

435 phenomena. In the following parts, the model validity is first discussed. Then, the material

436 behaviour upon hydration in constant volume conditions as well as interesting contributions of

437 the DEM to interpretation of laboratory tests results and constitutive modelling using double

438 structure models are discussed.

439

440

\subsection{Model validity}

441

442 The validity of the modelling approach mainly depends on the following assumptions:

$443 \quad$ - $\quad$ the material remains granular upon suction decrease;

$444 \quad$ - the shape of the simulated beads does not induce significant difference of behaviour;

$445 \quad-\quad$ the contact laws are valid;

446 - pellet hydration can be simulated by a radius increase.

448 Sample dismantling allowed the material granular nature to be observed upon hydration to

449 suction value as low as $4 \mathrm{MPa}$ (Figure 10a and 10b). Furthermore, the shape of the pellets was 
not notably modified. Only local deformation is observable at contact area. These deformations are irreversible.

452

In the simulations, granules are modelled as isotropic spheres. Their real shape is shown to remain nearly identical upon hydration (Figure 10a and 10b), but is characterised by a cylindershaped part between two spherical ends (Figure $1 \mathrm{a}$ and $1 \mathrm{~b}$ ). Pellets are nonetheless characterised by an aspect ratio (total height to diameter ratio) equal to 1 and considered subspherical. Wiacek et al. (2012) [42] showed that uniaxial compression of subspherical pea seeds could effectively be modelled by spheres in DEM. Furthermore, [36] show small differences between axial and radial values of elastic modulus for pellets within the investigated suction range. It is thus thought that isotropic beads are a suitable choice to model these bentonite pellets.

462

463 Assuming linear elasticity, the normal force-deflection relationship at contact is described by the

464 Hertz law (equations (8) and (9)) which had been shown to satisfactorily reproduce the experimental pellet force-deflection relationship [36]. It is proposed to include the influence of grain failure in the model to avoid reaching unrealistically high normal force values. It is assumed that failure initiates at contact and does not depend on the grain stress state. The pellet strength in the granular assembly is thus assumed to be described by the empirical equation proposed for free swelling conditions (equation (4)). As suggested by Figure 10a and $10 \mathrm{~b}$, particle failure is more characterised by an irreversible deformation at contact or apparition of cracks than by a general crushing. In this respect, contact plasticity is thought to be an interesting way of modelling this phenomenon as it allows irreversible deflection to arise in simulations. Elastic-plastic contact modelling approach $[43,44]$ is thus preferred to grain

474 crushing modelling approach $[45,46]$ to take into account grain failure. Compared to the 475 framework introduced by Thornton and Ning (1998) [43], the granule elastic limit in the present 476 study is given in term of a "limiting contact force", given by $R_{A}$, directly obtained from experimental results [36], instead of a "limiting contact pressure". Pellet failure in the simulation is thus taken into account by introducing perfect plasticity in the contact law, with the pellet 
strength decreasing upon hydration (equation (4)). The mean value of the ratio of total deflection to particle diameter using this approach is lower than 0.07 .

However, it is worth mentioning that experimental results evidenced a decrease of normal force at failure (constant suction) [36], which is not taken into account by the perfect plasticity of the contact behaviour adopted in the present study. Nevertheless, the calculated swelling pressure for suctions at which most of the contacts had reached the elastic limit appeared to be in the right order of magnitude.

Modelling the swelling of spherical particles within a granular assembly by a radius increase has already been performed in DEM, for super absorbent polymer hydration [35] or thermal expansion effect in granular materials [33, 34]. In these works, a water/heat transfer was introduced and the particles would swell accordingly. In the present work, the approach is slightly different because no transfer law is introduced. All particles swell simultaneously, suction is calculated from equation (3) and all mechanical parameters vary accordingly. It is based on the assumption that vapour diffusion in inter-pellet porosity occurs significantly faster than absorption of water by pellets. The macropores are thought to be full of air at the same relative humidity. From Figure 10a and 10b, it can be observed that the macroporosity is still clearly observable at $4 \mathrm{MPa}$ of suction. In addition, humid air is free to enter the sample macroporosity from either the bottom or the top of the cell (Figure 3). It is thus thought that the suction value imposed to all granules is identical within the isochoric cell. In addition, it is also suggested that there is still free volume available for the pellets to swell.

502 The model is able to reproduce the general "two phases" trend of the swelling pressure

503 development observed in the laboratory (DEM1 in Figure 9). The first phase, characterised by a 504 sharp increase of swelling pressure until a peak value is reached, is however overestimated by 505 the model. The overestimation of the swelling pressure at high suction in simulation is a consequence of several features: the modelled beads are all identically shaped (perfect spheres

507 of same diameter), cannot rearrange as a consequence of the small cell size $\left(H_{\text {cell }} / a_{\text {eq }}=4\right)$, 508 and have the same mechanical properties. 
510 It is highlighted in [36] that the variability of pellet mechanical properties is more important at

511 high suction. According to equation (8), the contact force is proportional to the pellet stiffness if

512 all pellets have the same stiffness. In case of a stiffness variability between two pellets in

513 contact, the term $E_{m} / 1-v^{2}$ in equations (8) would be given by [37]:

514

$$
\frac{E_{m}}{1-v^{2}}=\frac{1}{\frac{1-v_{1}^{2}}{E_{m 1}}+\frac{1-v_{2}^{2}}{E_{m 2}}}
$$

516 Where $E_{m 1}, E_{m 2}, v_{1}$ and $v_{2}$ are the Young modulus of pellet 1 and pellet 2 and the Poisson ratio

517 of pellet 1 and 2 in the contact. Equation (17) highlights that the contact stiffness would be

518 significantly smaller if the Young modulus of one of the pellets in contact were smaller.

520 The second phase, at lower suction, and lower variability of the mechanical properties, is closer

521 to experimental results. During the second phase, the initial features of the granular assembly

522 have less influence on the macroscopic response because contact forces have reached the

523 elastic limit and the swelling pressure mainly depends on the pellet strength and stiffness

524 decreases upon suction decrease. Thus, swelling pressures in DEM1, DEM2, and DEM3 have

525 comparable values. "Two phases" trends had already been obtained for Febex granular

526 bentonite $[19,30]$. In these studies the swelling pressure would increase then remain nearly

527 constant until final saturation (zero suction). It was also suggested that the "plateau" swelling

528 pressure value was a consequence of the pellet strength and stiffness decrease upon hydration.

530 In the present study, pellet initial diameter is $7 \mathrm{~mm}$. To model pellets of a different size, the

531 simulation method can directly be applied as long as the aforementioned conditions for model

532 validity are verified. Pellet mechanical properties are likely to be influenced by the pellet size

533 [36]. These latter should thus be carefully determined before modelling pellets of different size. 
537 Wang et al. (2012) [8] proposed an estimation of the final swelling pressure of a compacted

538 bentonite-based material from its initial dry density. From this estimation, the final swelling

539 pressure of the studied material is $\sim 0.21 \mathrm{MPa}$, higher than the "plateau" value obtained in the

540 swelling pressure tests. The swelling pressure is thus expected to increase upon further suction

541 decrease. Compacted bentonite materials display a change in the hydration and swelling

542 mechanism at low suction. Saiyouri et al. (2004) [47] evidenced that the water uptake

543 mechanisms of compacted MX80 bentonite materials undergo a significant change between $\sim 3$

544 and $\sim 7 \mathrm{MPa}$ of suction. This suction range coincides with a sharp irreversible decrease of the

545 number of layers by clay particle. The proportion of interparticle porosity increases, diffuse

546 double layers are thought to develop and the proportion of interparticle water considerably

547 increases. This is consistent with the transition from the microstructural domain to a dry density-

548 dependent domain of the water retention curve of MX80-based materials [4] and the pore

549 volume reorganisation of MX80 pellets [48].

550

551 The DEM model would be unable to reproduce this behaviour and is considered not to be

552 appropriate to describe the swelling pressure evolution upon suction decrease below this

553 threshold (between $\sim 3$ and $\sim 7 \mathrm{MPa}$ of suction), even if the material remains granular below

554 these values (Figure 10a and 10b).

555

556 5.3. Influence of walls on the measured response

557

558 Vargas and McCarthy (2007) [30] performed simulations of particles swelling in both a fixed-

559 walls and a free moving-walls cell. In the first case, particle swelling induced an increase of

560 contact force instead of particle rearrangement. In the second case, the mean contact force was

561 not significantly increasing upon particle radius increase. These two extreme cases highlight

562 that the more particles rearrange, the more the coordination number will increase and the less

563 contact forces will.

564

565 The evolution of the mean elastic normal deflection upon radius increase, at contact between

566 pellets and the upper wall, is presented in Figure 16. DEM1 results (optimised preparation and 
567 friction during hydration) are characterised by a nearly perfect linear deflection-radius variations

568 relationship until reaching the peak swelling pressure. The linear relationship between these two

569 parameters is close to the " $\Delta \delta_{N}{ }^{e}=\Delta R$ " line which implies negligible rearrangement of the

570 granular assembly upon swelling. The swelling pressure increase is thus significant and elastic

571 limit in contacts with the upper wall is reached almost simultaneously, resulting in an

572 overestimated peak value and an accentuated transition between phases 1 and 2. This

573 tendency towards deflection increase instead of particle rearrangement in the simulations is

574 also underlined in Figure 16 by the significant increase of the mean elastic normal deflection

575 after the cell closure in DEM2 (friction during preparation). The considered solid fraction (0.553)

576 is low and these features should not be observed in larger granular assemblies, less influenced

577 by walls. Since pellet-wall contacts are stiffer than pellet-pellet contacts, negligible

578 rearrangements also explain the more significant increase in contact plasticity at contact with

579 walls compared to the mean increase in the samples. As a consequence, the post-peak

580 decrease of swelling pressure can be overestimated if swelling pressure is measured at contact

581 with the wall instead of calculating the mean stress in the sample (Figure 17).

582

583 The influence of the walls is furthermore underlined by the coordination number following

584 preparation (Figure 12): the coordination number of frictionless bead assemblies was 5.4

585 instead of the bulk value $\sim 6$ [41].

586

587 These results suggest that the variability associated to sample preparation stems from wall

588 influence. Experimental tests are influenced by the walls as well, because they share the same

589 solid fraction and $H_{c e l l} / a_{e q} 0$ ratio.

590

591 5.4. Contribution of DEM to laboratory testing

592

593 Thanks to the DEM ability to model the wall-pellet interaction and access grain-scale

594 phenomena, simulation results can provide an insight into swelling pressure tests carried out at

595 laboratory scale, especially since walls have non-negligible influence on the measured

596 response. 
598 The influence of sample preparation on test results was highlighted by DEM simulations (Figure

599 9). The general trend of DEM2 results suggests that initial pressure required to close the cell

600 before hydration can cause a significant increase of plastic contact proportion (Figure 11b). As a

601 consequence, hydration phase 1 appeared very limited in DEM2. The sample behaviour was

602 mainly controlled by equations (2) and (4) and the number of contacts between pellets and the

603 upper wall, rather than the particle swelling. Even if plasticity does not arise during the closure

604 step, closure can have an influence on the behaviour upon hydration. At granular state, the

605 mean stress in the sample is proportional to the product of coordination number and mean

606 contact force. It was highlighted in Figure 12 that the evolution of the coordination number was

607 not influenced by the sample preparation. Thus, samples prepared under higher initial closure

608 pressure are likely to reach plasticity more rapidly. This is actually suggested by SP1 and SP2

609 test results. It is recommended that the control of the initial state is as important as the value of

610 the sample density to study the swelling pressure development of pellet materials.

612 Beside variability arising from wall influence, small pressure sensors in the laboratory have

613 been shown to induce additional variability of the measured swelling pressure. DEM allowed the

614 influence of the pressure sensor diameter on the variability of the measured swelling pressure

615 to be quantified. This variability, along with the variability of behaviour associated to the sample

616 small size, increases the difficulty to study the intrinsic behaviour of pellet mixtures at laboratory

617 scale. For granules with the same mechanical behaviour, the coefficient of variation of swelling

618 pressure obtained from simulations thus depends on both the preparation in a small cell and the

619 ratio of sensor diameter to pellet diameter.

620

621 It was discussed that an overestimation of the calculated swelling pressure can arise in the

622 elastic phase of the granule swelling from some of the model simplifications, especially at the

623 highest suction values. However, concerning the variability of this measure, these

624 simplifications can reduce the coefficient of variation. Owing to the dispersion of the granule real

625 mechanical properties at high suctions, the calculated coefficient of variation associated to

626 swelling pressure is likely to be underestimated by the model in the elastic part of the hydration 
627 path. As contact forces reach the elastic limit, normal forces reach less dispersed values and 628 the calculated coefficient of variation decreases.

629

630 These results are of importance, since most of the time the material behaviour is studied at

631 laboratory scale, with inevitable constraints concerning the number and sizes of sensors,

632 related to the cell size. It is recommended that experiments performed to determine material

633 parameters for modelling purpose should be interpreted carefully considering the order of

634 magnitude of the variability.

635

636 Simulation results highlighted the influence of interactions at contacts between pellets on the

637 material behaviour upon hydration. Since pellets would lose their structure upon full saturation,

638 the material behaviour is sensitive to the hydration method at laboratory scale. The influence of

639 the granular nature of the material is likely to be underestimated upon fast liquid water

640 hydration.

641

642 5.5. Contribution of DEM to constitutive modelling of pellet materials

643

644 The proposed DEM model has been shown to reproduce the main features of the material

645 behaviour upon partial hydration in constant-volume conditions. The relationships between the

646 behaviour of the granules and the macrostructural behaviour can be identified. It can provide

647 valuable information for the modelling of pellet mixture behaviour using double structure

648 models. This modelling framework has been used in previous studies to model pellet materials

649 upon hydration, considering the pellet phase as the microstructure $[20,21,23]$.

650

651 For this purpose, DEM can be a complementary tool to experimental studies. It was highlighted 652 that results of experimental tests performed at the laboratory scale on pellet mixtures can be 653 characterised by a significant variability. This latter can make the determination of the material 654 intrinsic behaviour difficult. DEM allows uncertainty and variability to be taken into account, at 655 low simulation cost. DEM model parameters can be determined from experimental tests at the 
656 pellet scale [36]. Then, the model can be validated against swelling pressure tests or other 657 common tests at the laboratory scale.

658

659 Simulations may be performed in conditions where the intrinsic behaviour of the material can be

660 addressed. DEM could help determining constitutive laws for the mechanical behaviour of a 661 pellet assembly and relationship between pellet swelling and pellet assembly swelling. In this

662 respect, a perspective arising from the present study results is to perform DEM simulations of 663 large pellet assemblies of different solid fractions upon hydration, avoiding the influence of wall

664 and small sample sizes. In this way, constitutive laws describing the material before homogenisation could be proposed and integrated in a double structure model.

666

667

668

\section{Conclusions}

669

The hydromechanical behaviour of pellet assemblies was studied through laboratory and DEMsimulated swelling pressure tests. DEM model parameters were obtained from pellet scale experimental characterisation. The DEM model was validated against experimental results.

673 Numerical results allowed the experimental results to be interpreted with respect to grain scale 674 phenomena.

675

676 Compared to traditional compacted powder materials, pellet materials are characterised by an initial granular nature, which was shown in the present study to control the material behaviour

678 upon hydration to suction as low as $4 \mathrm{MPa}$. In particular, it was shown that, in constant volume conditions, the granule strength and stiffness control the macroscopic response. Upon

680 hydration, the swelling pressure develops in two phases. The first is characterised by an elastic 681 increase of the normal contact forces and is affected by the initial state of the material, thus the 682 sample preparation in laboratory tests. The second is characterised by the occurrence of 683 contact plasticity and is less affected by the initial state.

684 
DEM simulations satisfactorily reproduced the material behaviour observed at laboratory scale.

686 DEM results suggest that the $30 \mathrm{~mm} \times 60 \mathrm{~mm}$ isochoric cell $\left(H_{c e l l} / a_{e q 0}=4\right)$ was not a true

687 representative elementary volume, due to its small size and the significant influence of the

688 walls. In addition, it was shown that a low value of the ratio of sensor diameter to pellet diameter

689 induces significant measurement variability in the investigated suction range.

690

691 A perspective arising from the present study is the DEM modelling of large pellet assemblies

692 upon hydration, with no influence of the walls. Constitutive laws for the material before

693 homogenisation could be proposed and provide an interesting framework for further FEM

694 simulations of the entire hydration path, using double structure models in which pellets could be

695 considered as the microstructural phase.

696

697

698 Conflict of Interest:

699

700 The authors declare that they have no conflict of interest.

701

702

703

\section{References}

704

705

1. Blatz JA, Graham J, Chandler NA (2002) Influence of suction on the strength

706 and stiffness of compacted sand\&\#150;bentonite. Can Geotech J 39:1005-1015.

707 https://doi.org/10.1139/t02-056

708

2. Lloret A, Villar M V, Sanchez M, et al (2003) Mechanical behaviour of heavily

709 compacted bentonite under high suction changes. Geotechnique 53:27-40.

710 https://doi.org/10.1680/geot.53.1.27.37258

3. Agus SS, Schanz T (2005) Effect of Shrinking and Swelling on Microstructures

712 and Fabric of a Compacted Bentonite-Sand Mixture. Proc Int Conf Probl Soils, 25-27 May 2005,

713 East Mediterr Univ Famagusta, N Cyprus 
4. Villar M V. (2007) Water retention of two natural compacted bentonites. Clays Clay Miner 55:311-322. https://doi.org/10.1346/CCMN.2007.0550307

5. Saiyouri N, Hicher PY, Tessier D (2000) Microstructural approach and transfer water modelling in highly compacted unsaturated swelling clays. Mech Cohesive-frictional Mater $718 \quad 5: 41-60$ bentonite: a microstructure approach. Géotechnique 56:291-304. https://doi.org/10.1680/geot.2006.56.5.291

7. Tang, AM, Cui YJ, Le TT (2008) A study on the thermal conductivity of compacted bentonites. Appl Clay Sci 41:181-189

8. Wang Q, Tang AM, Cui YJ, et al (2012) Experimental study on the swelling behaviour of bentonite/claystone mixture. Eng Geol 124:59-66. https://doi.org/10.1016/j.enggeo.2011.10.003

9. Saba S, Cui Y, Tang AM, Barnichon J (2014) Investigation of the swelling behaviour of compacted bentonite-sand mixtures by mock-up tests. Can Geotech J 51:13991412

10. Sun H, Mašín D, Najser J, et al (2019) Bentonite microstructure and saturation evolution in wetting-drying cycles evaluated using ESEM, MIP and WRC measurements. Geotechnique 69:713-726. https://doi.org/10.1680/jgeot.17.P.253

734 microstructural evolution of MX-80 bentonite during wetting and drying cycles. Géotechnique 64:721-734. https://doi.org/10.1680/geot.14.P.017

12. Massat L, Cuisinier O, Bihannic I, et al (2016) Swelling pressure development 737 and inter-aggregate porosity evolution upon hydration of a compacted swelling clay. Appl Clay

738 Sci 124-125:197-210. https://doi.org/10.1016/j.clay.2016.01.002

13. Keller LM, Seiphoori A, Gasser P, et al (2014) The pore structure of compacted

740 and partly saturated MX-80 bentonite at different dry densities. Clays Clay Miner 62:174-187. https://doi.org/10.1346/CCMN.2014.0620302

14. Komine H, Ogata N (2003) New equations for swelling characteristics of 743 bentonite-based buffer materials. Can Geotech J 40:460-475. https://doi.org/10.1139/t02-115 
15. Cuisinier O, Masrouri F (2005) Hydromechanical behaviour of a compacted swelling soil over a wide suction range. Eng Geol 81:204-212. https://doi.org/10.1016/j.enggeo.2005.06.008

16. Karnland O, Nilsson $\mathrm{U}$, Weber $\mathrm{H}$, Wersin $\mathrm{P}(2008)$ Sealing ability of Wyoming bentonite pellets foreseen as buffer material - Laboratory results. Phys Chem Earth 33:472475. https://doi.org/10.1016/j.pce.2008.10.024

17. Volckaert, G., Bernier, F., Alonso, E. E., et al (1996). Thermal-hydraulicmechanical and geochemical behaviour of the clay barrier in radioactive waste repositories (model development and validation), EUR 16744 EN. Luxembourg: Publications of the European Communities.

18. van Geet M, Volckaert G, Roels S (2005) The use of microfocus X-ray computed tomography in characterising the hydration of a clay pellet/powder mixture. Appl Clay Sci 29:73-87. https://doi.org/10.1016/j.clay.2004.12.007

19. Hoffmann C, Alonso EE, Romero E (2007) Hydro-mechanical behaviour of bentonite pellet mixtures. Phys Chem Earth 32:832-849. https://doi.org/10.1016/j.pce.2006.04.037

20. Alonso EE, Romero E, Hoffmann C (2011) Hydromechanical behaviour of compacted granular expansive mixtures: experimental and constitutive study. Géotechnique 61:329-344. https://doi.org/10.1680/geot.2011.61.4.329

21. Gens A, Valleján B, Sánchez M, et al (2011) Hydromechanical behaviour of a

764 heterogeneous compacted soil: experimental observations and modelling. Géotechnique 765 61:367-386. https://doi.org/10.1680/geot.SIP11.P.015

766 22. Molinero-Guerra A, Mokni N, Delage P, et al (2017) In-depth characterisation of 767 a mixture composed of powder/pellets MX80 bentonite. Appl Clay Sci 135:538-546.

768 https://doi.org/10.1016/j.clay.2016.10.030

23. Mokni N, Molinero-Guerra A, Cui Y-J, et al (2019) Modelling the long-term

770 hydro-mechanical behaviour of a bentonite pellet/powder mixture with consideration of initial structural heterogeneities. Géotechnique 1-61. https://doi.org/10.1680/igeot.18.p.110 

mixtures over a wide suction range. Eng Geol 264:105383. https://doi.org/10.1016/j.enggeo.2019.105383

Liu Z, Ye W, Zhang Z, et al (2019) Particle size ratio and distribution effects on

776

777

778

779

780

781

782

783

784

785

786

787

788

789

790

791

792

793

794

795

796

797

798

799

packing behaviour of crushed GMZ bentonite pellets. Powder Technol 351:92-101.

https://doi.org/10.1016/j.powtec.2019.03.038

26. Liu, Z., Cui, Y., Ye, W. et al (2020) Investigation of the hydro-mechanical behaviour of GMZ bentonite pellet mixtures. Acta Geotech. https://doi.org/10.1007/s11440-02000976-y

27. Molinero-Guerra A, Aimedieu P, Bornert M, et al (2018) Analysis of the structural changes of a pellet/powder bentonite mixture upon wetting by X-ray computed microtomography. Appl Clay Sci 165:164-169. https://doi.org/10.1016/j.clay.2018.07.043

28. Yigzaw ZG, Cuisinier O, Massat L, Masrouri F (2016) Role of different suction components on swelling behavior of compacted bentonites. Appl Clay Sci 120:81-90. https://doi.org/10.1016/j.clay.2015.11.022

29. Imbert C, Villar MV (2006) Hydro-mechanical response of a bentonite pellets/powder mixture upon infiltration. Appl Clay Sci 32:197-209. https://doi.org/10.1016/j.clay.2006.01.005

30. Molinero Guerra A, Cui YJ, Mokni N, et al (2018) Investigation of the hydromechanical behaviour of a pellet/powder MX80 bentonite mixture using an infiltration column. Eng Geol 243:18-25. https://doi.org/10.1016/j.enggeo.2018.06.006

31. Alonso EE, Hoffmann C, Romero E (2010) Pellet mixtures in isolation barriers. J Rock Mech Geotech Eng 2:12-31. https://doi.org/10.3724/SP.J.1235.2010.00012

32. Radjai, F. \& Dubois, F. (2011). Discrete-element modeling of granular materials. Wiley-ISTE, ISBN 978-1-84821-260-2.

33. Vargas WL, McCarthy JJ (2007) Thermal expansion effects and heat conduction in granular materials. Phys Rev E - Stat Nonlinear, Soft Matter Phys 76:1-8. https://doi.org/10.1103/PhysRevE.76.041301 

investigation on thermally-induced shakedown of granular materials. Granul Matter 19:1-11. https://doi.org/10.1007/s10035-016-0690-5

35. Sweijen T, Chareyre B, Hassanizadeh SM, Karadimitriou NK (2017) Grain-

804 scale modelling of swelling granular materials; application to super absorbent polymers. Powder Technol 318:411-422. https://doi.org/10.1016/j.powtec.2017.06.015

806

36.

Darde B, Tang AM, Pereira J-M, et al (2018) Hydro-mechanical behaviour of high-density bentonite pellet on partial hydration. Géotechnique Lett 8:330-335.

808 https://doi.org/10.1680/jgele.18.00114

37. Johnson KL (1985) Contact Mechanics. Cambridge University Press,

810 Cambridge, UK

811 38. Darde B, Tang AM, Roux JN, et al (2020) Effects of the initial granular structure

812 of clay sealing materials on their swelling properties : experiments and DEM simulations. EPJ

813 Nuclear Sci. Technol. 6. https://doi.org/10.1051/epjn/2019059.

814 39. Tang A-M, Cui Y-J (2005) Controlling suction by the vapour equilibrium

815 technique at different temperatures and its application in determining the water retention

816 properties of MX80 clay. Can Geotech J 42:287-296. https://doi.org/10.1139/t04-082

$817 \quad$ 40. Agnolin I, Roux JN (2007) Internal states of model isotropic granular packings.

818 I. Assembling process, geometry, and contact networks. Phys Rev E - Stat Nonlinear, Soft

819 Matter Phys 76:1-27. https://doi.org/10.1103/PhysRevE.76.061302

820 41. Roux J-N (2000) Geometric origin of mechanical properties of granular

821 materials. Phys Rev E - Stat Nonlinear, Soft Matter Phys 61:1-35

822 42. Wiacek J, Molenda M, Horabik J, Ooi JY (2012) Influence of grain shape and

823 intergranular friction on material behavior in uniaxial compression: Experimental and DEM

824 modeling. Powder Technol 217:435-442. https://doi.org/10.1016/j.powtec.2011.10.060

825 43. Thornton C, Ning Z (1998) A theoretical model for the stick/bounce behaviour of 826 adhesive, elastic- plastic spheres. Powder Technol 99:154-162. https://doi.org/10.1016/S0032$827 \quad 5910(98) 00099-0$ 
44. Thornton C, Cummins SJ, Cleary PW (2017) On elastic-plastic normal contact

829 force models, with and without adhesion. Powder Technol 315:339-346.

830 https://doi.org/10.1016/j.powtec.2017.04.008

831 45. Bolton MD, Nakata Y, Cheng YP (2008) Micro- and macro-mechanical

832 behaviour of DEM crushable materials. Géotechnique 58:471-480.

833 https://doi.org/10.1680/geot.2008.58.6.471

834 46. Cheng YP, Bolton MD, Nakata Y (2004) Crushing and plastic deformation of 835 soils simulated using DEM. Géotechnique 54:131-141.

836 https://doi.org/10.1680/geot.2004.54.2.131

837 47. Saiyouri N, Tessier D, Hicher PY (2004) Experimental study of swelling in

838 unsaturated compacted clays. Clay Miner 39:469-479.

839 https://doi.org/10.1180/0009855043940148

840 48. Molinero-Guerra A, Delage P, Cui Y-J, et al (2019) Water retention properties

841 and microstructure changes of a bentonite pellet upon wetting/drying; application to radioactive

842 waste disposal. Géotechnique 1-41. https://doi.org/10.1680/jgeot.17.p.291

843

844 
$847 a_{e q} 0$ : initial sphere diameter in the simulations

$848 a_{e q} i$ : sphere diameter at step $i$ in the simulations

$849 a_{e q}$ : sphere diameter in the simulations

$850 \quad C_{A}$ : model parameter

$851 D_{0}$ : initial diameter of the pellet

$852 D_{\text {sensor: }}$ diameter of the pressure sensor

$853 E_{m}$ : pellet Young modulus

$854 F_{\max }$ : maximum normal force on a particle

$855 \quad F_{N}$ : normal contact elastic force

$856 F_{\text {net }}$ : net force on a particle

$857 \quad F_{T}$ : norm of the tangential reaction vector

$858 \boldsymbol{F}_{\boldsymbol{T}}$ : tangential reaction vector

$859 \dot{\boldsymbol{F}}_{T}$ : increment of tangential reaction vector

$860 h_{0}$ : initial height of the cylinder-shaped part of the pellet

$861 H_{0}$ : initial total height of the pellet

$862 H_{\text {cell }} 0$ : initial height of the cylinder

$863 H_{c e l l}$ : height of the cylinder during the swelling step

864 m: mean value

$865 m_{p}$ : mass of the pellet

$866 N_{c}$ : number of particle-particle contacts

$867 \quad N_{c 2}$ : number of particle-wall contacts

$868 N_{p}$ : number of particles

$869 N^{v}$ : viscous component of the contact force

$870 \quad p$ ': effective mean stress

$871 P_{S}^{*}$ : apparent swelling pressure at equilibrium in the simulations

$872 P_{S X P}$ : axial pressure in swelling pressure tests

$873 R_{A}$ : pellet strength

$874 R_{c}$ : curvature radius of the pellet

$875 s_{0}$ : initial suction of the pellet

$876 \quad V$ : volume of the pellet

$877 w_{0}$ : initial water content of the pellet 
$878 x_{c \text { sup }}^{p}$ : evolution of the mean plastic contacts proportion for contacts between pellets and the 879 upper wall

$880 x_{c t o t}^{p}$ : evolution of the mean plastic contacts proportion for the whole sample

881 Z: coordination number

$882 \alpha_{m}$ : model parameter

$883 \alpha_{v}$ : Damping parameter

$884 \beta_{m}$ : model parameter

$885 \Gamma_{\max }$ : maximum moment on a particle

$886 \Gamma_{\text {net }}$ : net moment on a particle

$887 \delta_{N}{ }^{e}$ : normal elastic deflection at contact

$888 \Delta \delta_{N}{ }^{e}:$ variation of the mean normal elastic deflection during particle swelling

$889 \Delta R$ : variation of the particle radius during swelling

$890 \quad \delta_{T}$ : relative tangential displacement vector

$891 \dot{\boldsymbol{\delta}}_{\boldsymbol{T}}$ : increment of relative tangential displacement vector

$892 \epsilon$ : tolerance value for equilibrium in the simulations

$893 \lambda_{i}$ : parameter describing the diameter increment at step i in DEM

$894 \lambda_{0}$ : parameter describing the diameter increment at initial state in DEM

$895 \lambda_{f}$ : parameter describing the diameter increment at final state in DEM

$896 \varepsilon_{V m}$ : pellet volumetric strain

$897 \mu$ : friction coefficient

$898 \quad v$ : pellet Poisson ratio

$899 \rho_{d 0}$ : initial dry density of the pellet

$900 \rho_{s}:$ MX80 particle density

$901 \sigma:$ standard deviation

902

903

904 
906

907

TABLE 1 Properties of the MX80 bentonite

908

\begin{tabular}{cc}
\hline Property & Value \\
\hline Particle density, $\rho_{\mathrm{s}}\left(\mathrm{g} / \mathrm{cm}^{3}\right)$ & 2.77 \\
\hline Smectite content $(\%)$ & 80 \\
\hline Liquid limit (\%) & 560 \\
\hline Plastic limit & 53 \\
\hline CEC (meq/g) & $98 / 100$ \\
\hline
\end{tabular}

909

910

911 TABLE 2 Initial properties of the granules

912

\begin{tabular}{cc}
\hline Property & Value \\
\hline Dry density, $\rho_{\mathrm{d} 0}\left(\mathrm{Mg} / \mathrm{m}^{3}\right)$ & 1.90 \\
\hline Water content, $\mathrm{w}_{0}(\%)$ & 12.2 \\
\hline Diameter, $\mathrm{D}_{0}(\mathrm{~mm})$ & 7.0 \\
\hline Height, $\mathrm{H}_{0}(\mathrm{~mm})$ & 7.0 \\
\hline Height of the cylinder-shaped part, $\mathrm{h}_{0}(\mathrm{~mm})$ & 5.0 \\
\hline Curvature radius, $\mathrm{R}_{\mathrm{c}}(\mathrm{mm})$ & 6.5 \\
\hline Suction, $\mathrm{s}_{0}(\mathrm{MPa})$ & 89 \\
\hline
\end{tabular}

913

914

915 
916 TABLE 3 Geometrical properties of isochoric cells

917

\begin{tabular}{cc}
\hline Property & Value \\
\hline Sample height, $H_{\text {cell }}(\mathrm{mm})$ & 30 \\
\hline Sample diameter, $D_{\text {cell }}(\mathrm{mm})$ & 60 \\
\hline Sensor diameter, $D_{\text {sensor }}(\mathrm{mm})$ & 30 \\
\hline Sample volume $\left(\mathrm{mm}^{3}\right)$ & 84823
\end{tabular}

918

919

920 TABLE 4 Suction values imposed using the vapour equilibrium technique

921

\begin{tabular}{cc}
\hline Salt solution & Suction $(\mathrm{MPa})$ \\
\hline $\mathrm{Mg}(\mathrm{NO} 3)_{2}$ & 82 \\
\hline $\mathrm{NH} 4 \mathrm{NO} 3$ & 59 \\
\hline $\mathrm{NaNO} 3$ & 40 \\
\hline $\mathrm{NaCl}$ & 38 \\
\hline$(\mathrm{NH} 4)_{2 \mathrm{SO}} 4$ & 25 \\
\hline $\mathrm{Na} 2 \mathrm{CO} 3$ & 13 \\
\hline $\mathrm{KNO} 3$ & 9 \\
\hline $\mathrm{K} 2 \mathrm{SO} 4$ & 4 \\
\hline
\end{tabular}

922

923

924 TABLE 5 Friction values used during preparation and hydration steps in DEM1, DEM2 and 925 DEM3 simulations. 100 simulations are performed for each type of simulation

926

927

Simulation $\mu$ (preparation step) (-) $\mu$ (swelling step) (-)

\begin{tabular}{ccc}
\hline DEM01 & 0 & 0.3 \\
\hline DEM02 & 0.3 & 0.3 \\
\hline DEM03 & 0 & 0 \\
\hline
\end{tabular}

928 
930

931

Fig. 1 Schematic view of a pellet

932

Height of the cylindershaped part, $h_{0}$

933

934

935

936

937

938

939

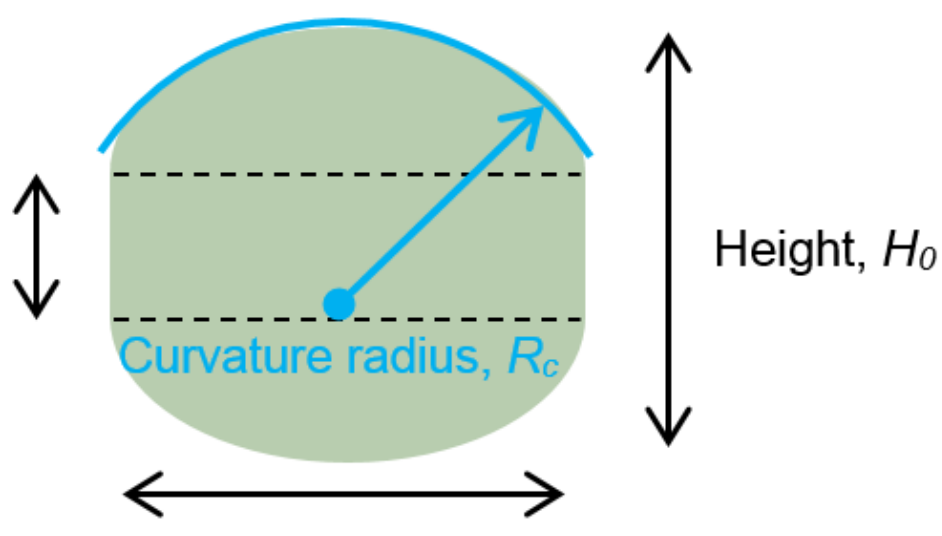

Diameter, $D_{0}$

Fig. 2 Isochoric cell used for swelling pressure tests

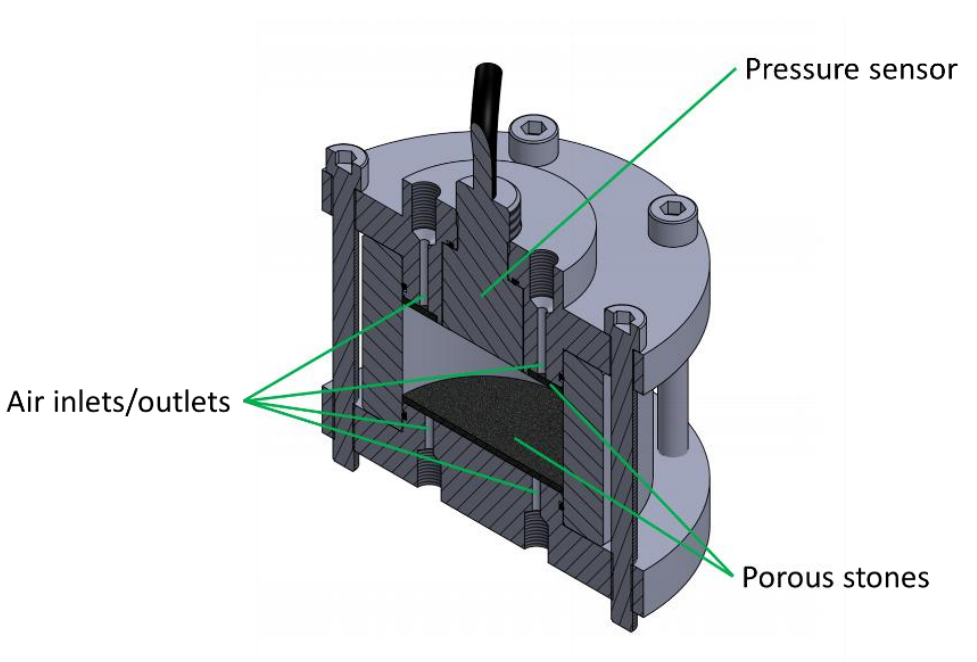


Fig. 3 Air circulation in the isochoric cell

941

942

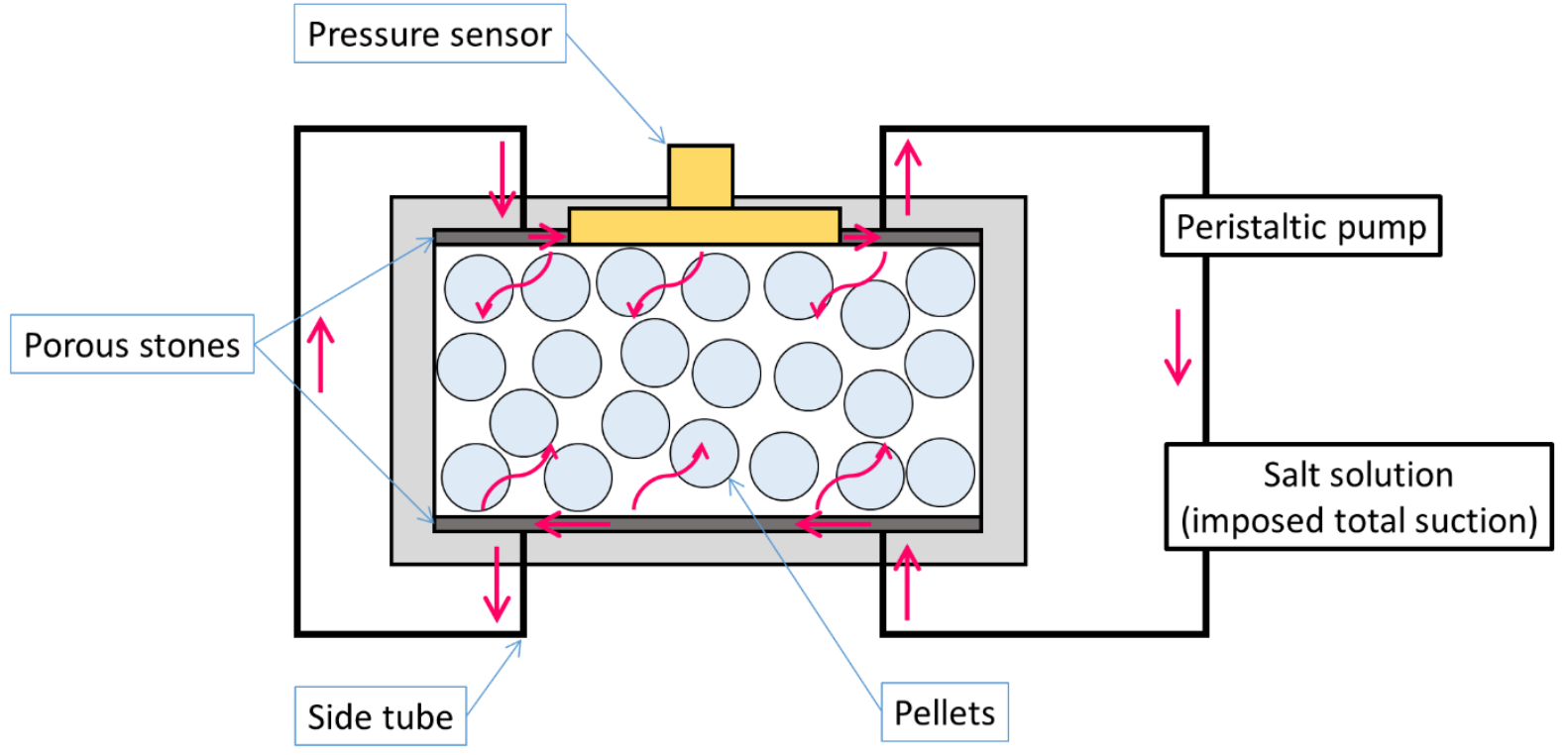

943

944 
946 Fig. 4a Hydromechanical behaviour of a pellet (Darde et al., 2018). a) Evolution of Volumetric 947 strain upon suction decrease for one pellet

948

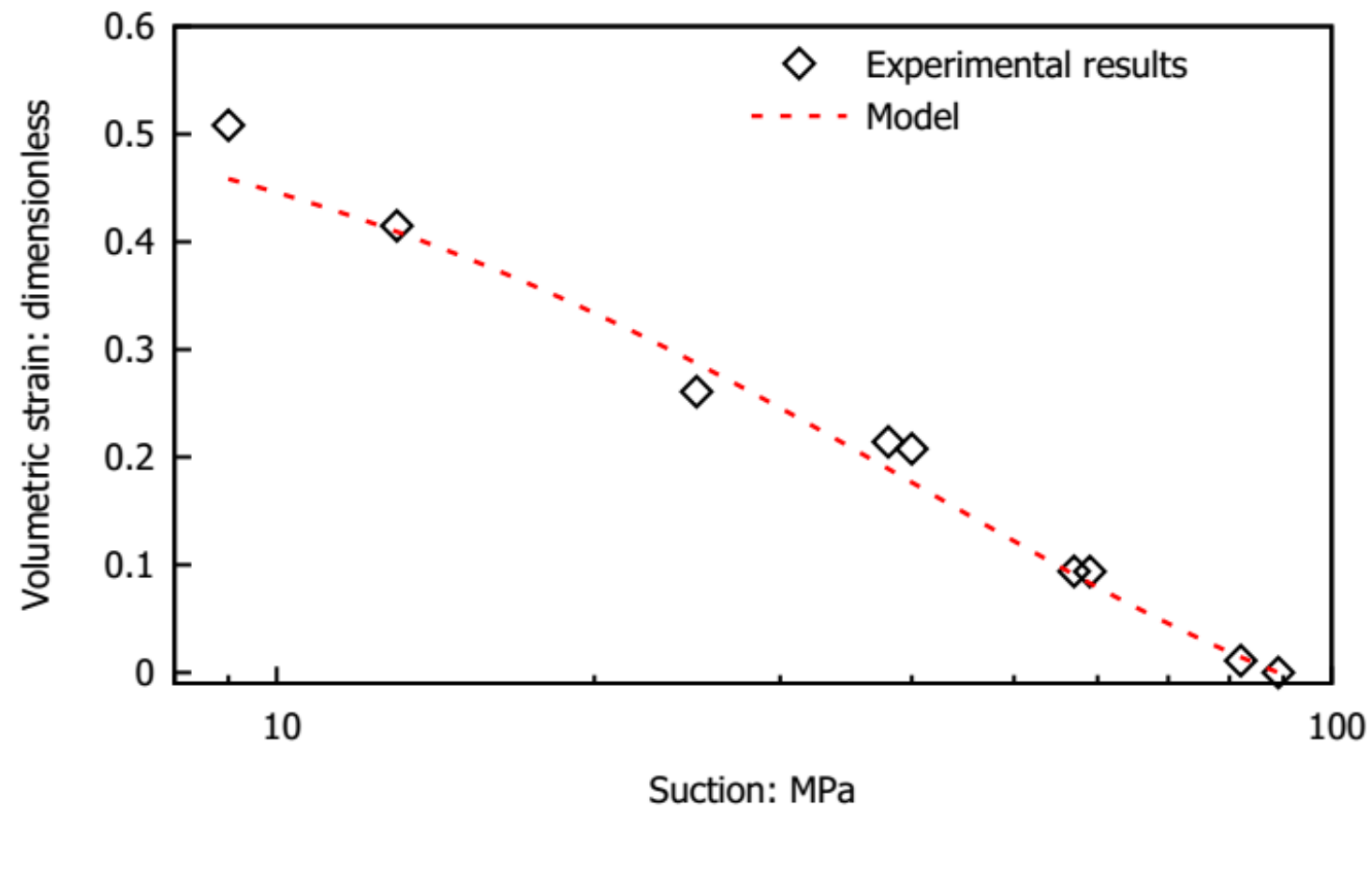

950 
951 Fig. 4b Evolution of Modulus and Strength upon suction decrease for one pellet

952

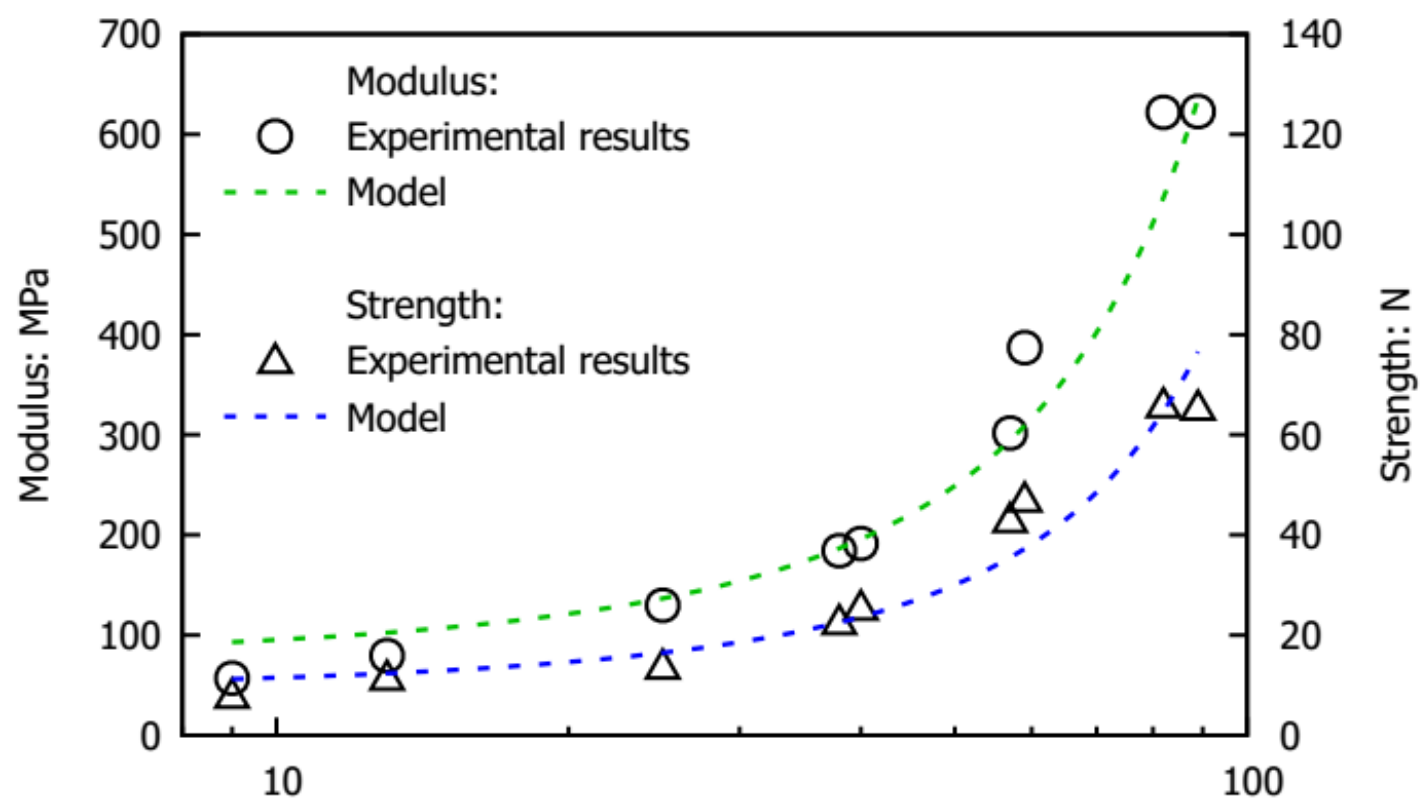

953

Suction: MPa

954

955

956 
957 Fig. 5 Contact between two particles in DEM

958

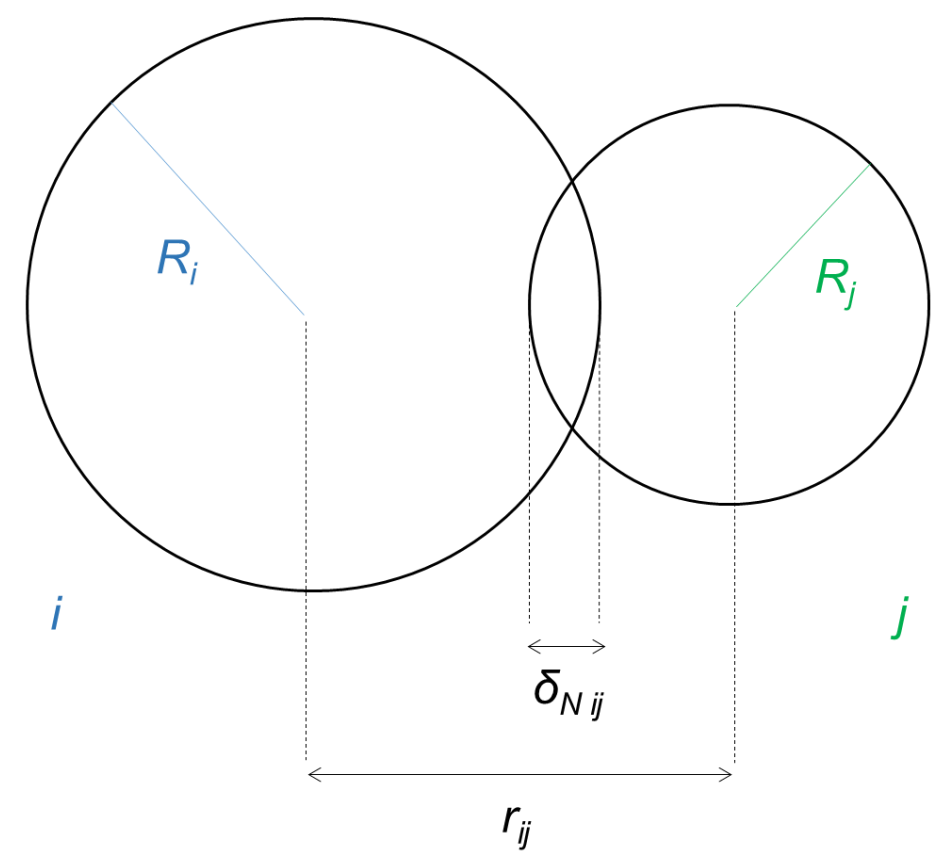

959

960

961

962 
963 Fig. 6a Contact laws used in the model: Normal reaction: Hertz law + perfect plasticity 964

965

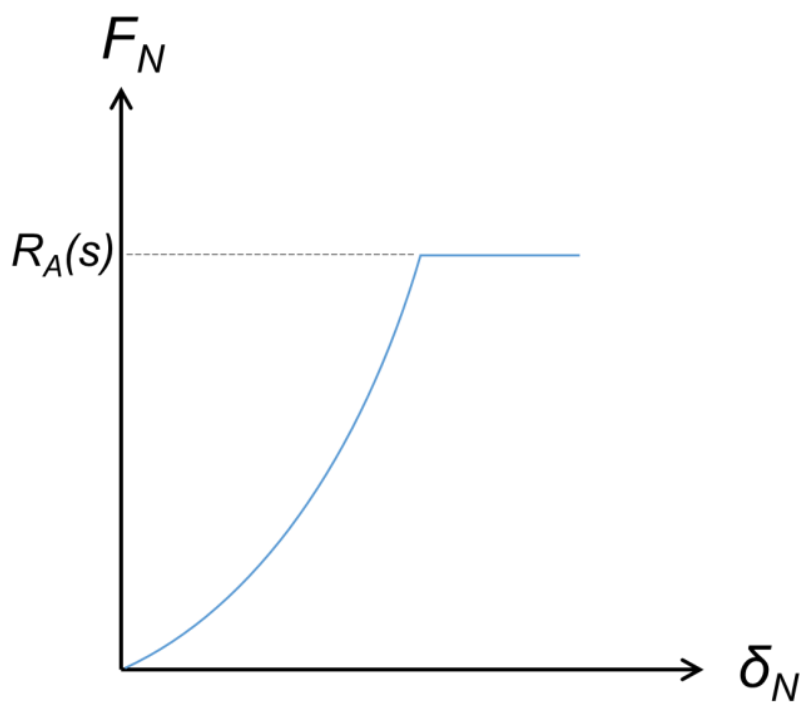

966

967 Fig $\mathbf{6 b}$ Tangential reaction: Simplified elasticity law + Coulomb friction

968

969

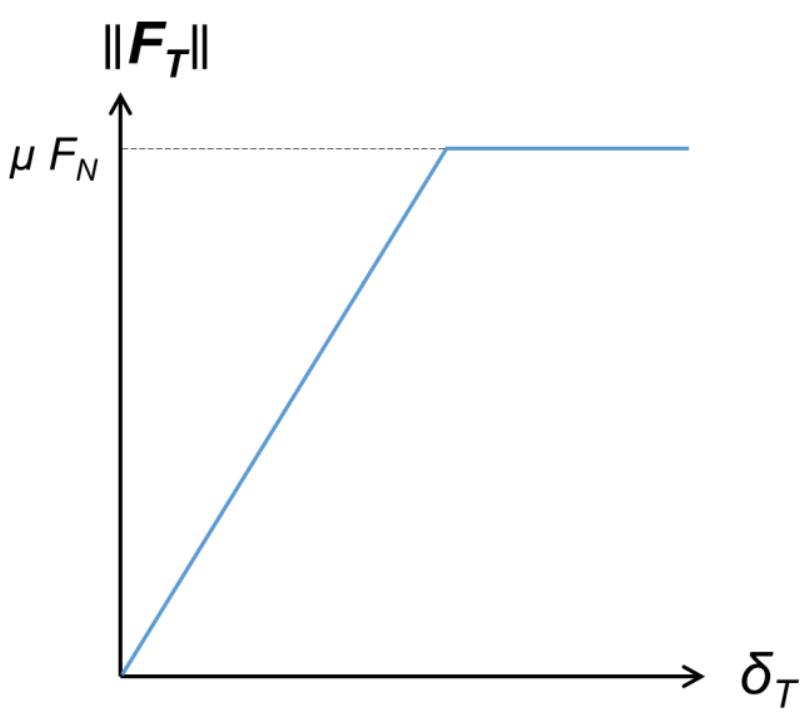

970

971

972 
973 Fig. 7a Preparation step in DEM

974

975

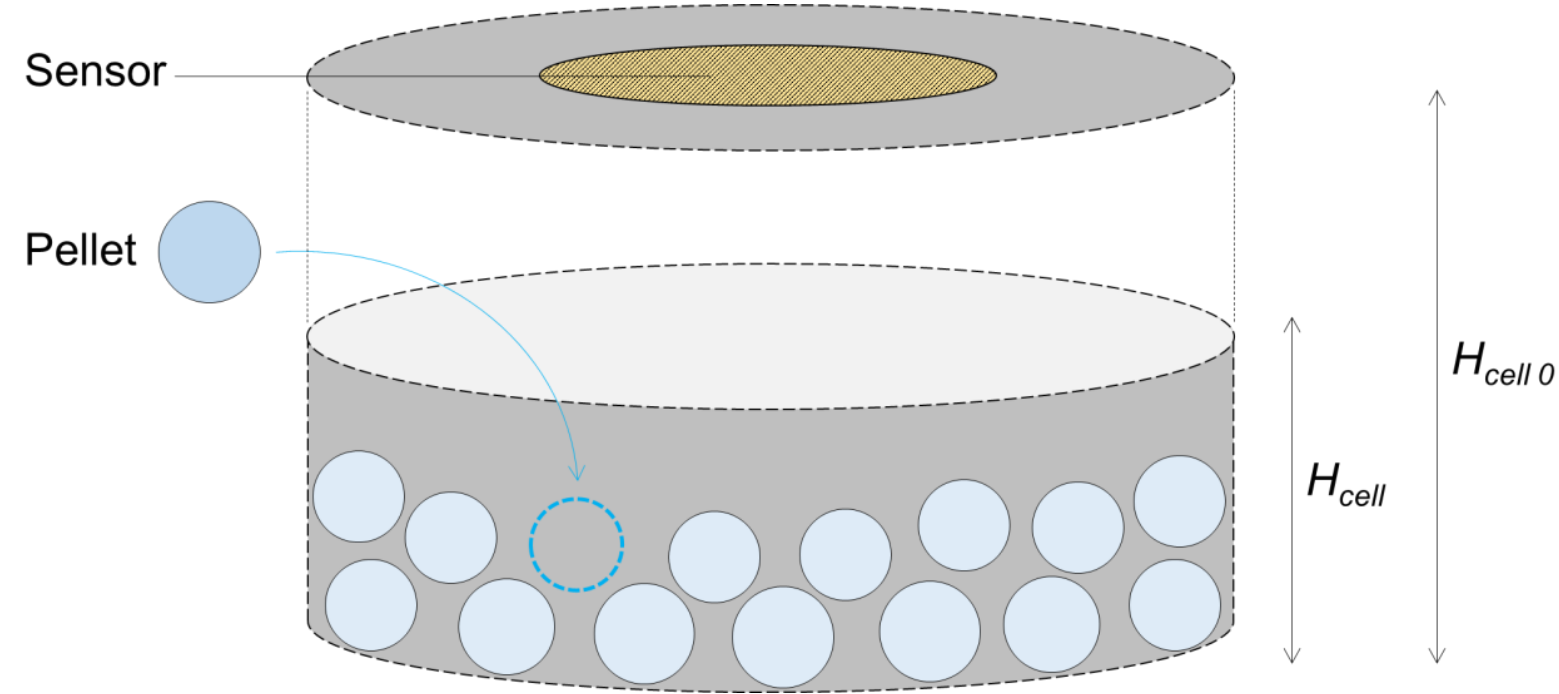

976

977

978 
979 Fig. 7b Dimensions of the isochoric cell

980

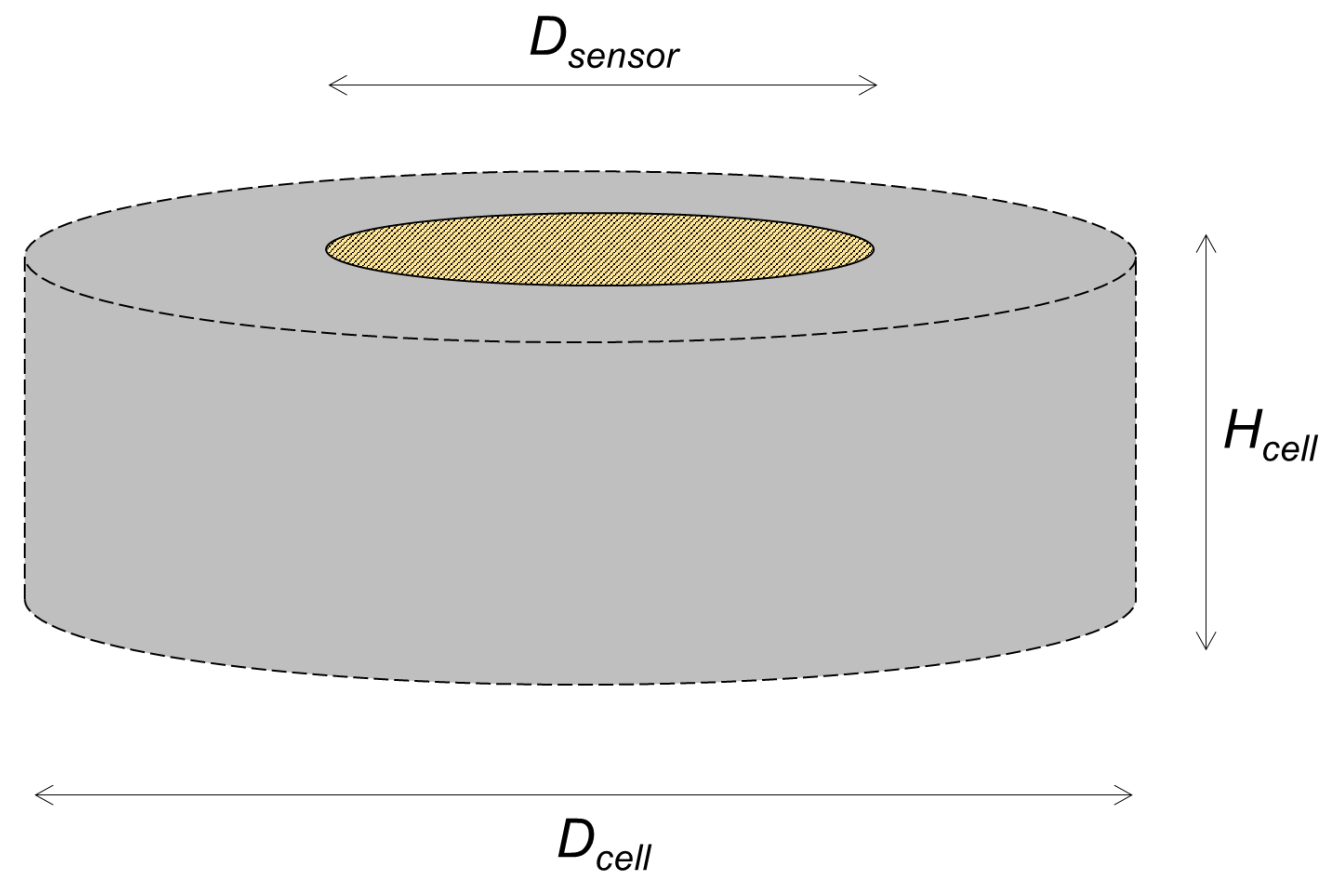

981

$D_{\text {pellet }}$

982

983

984 
986

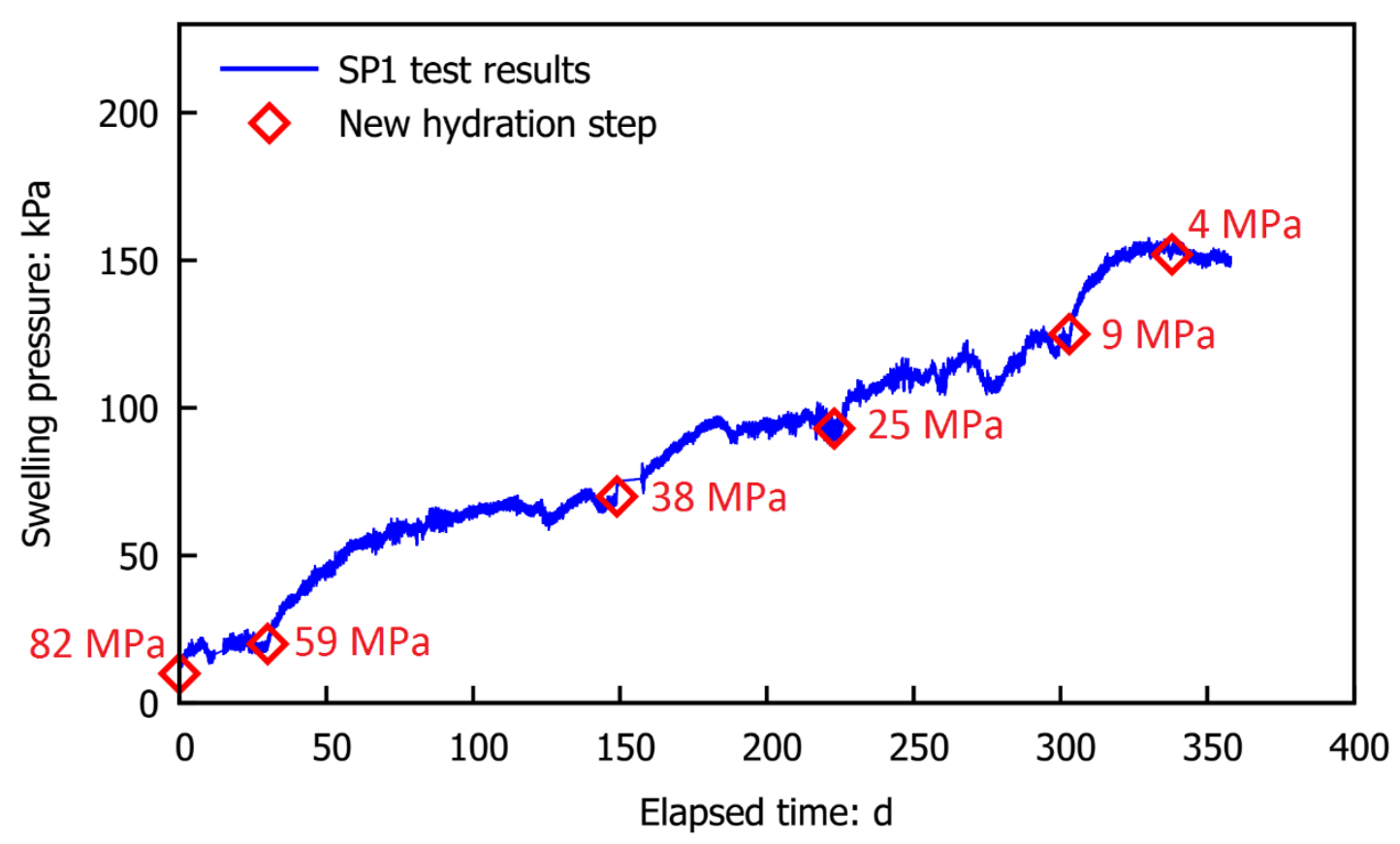

987

988

989

Fig. 8b Swelling pressure as a function of elapsed time for SP2

990

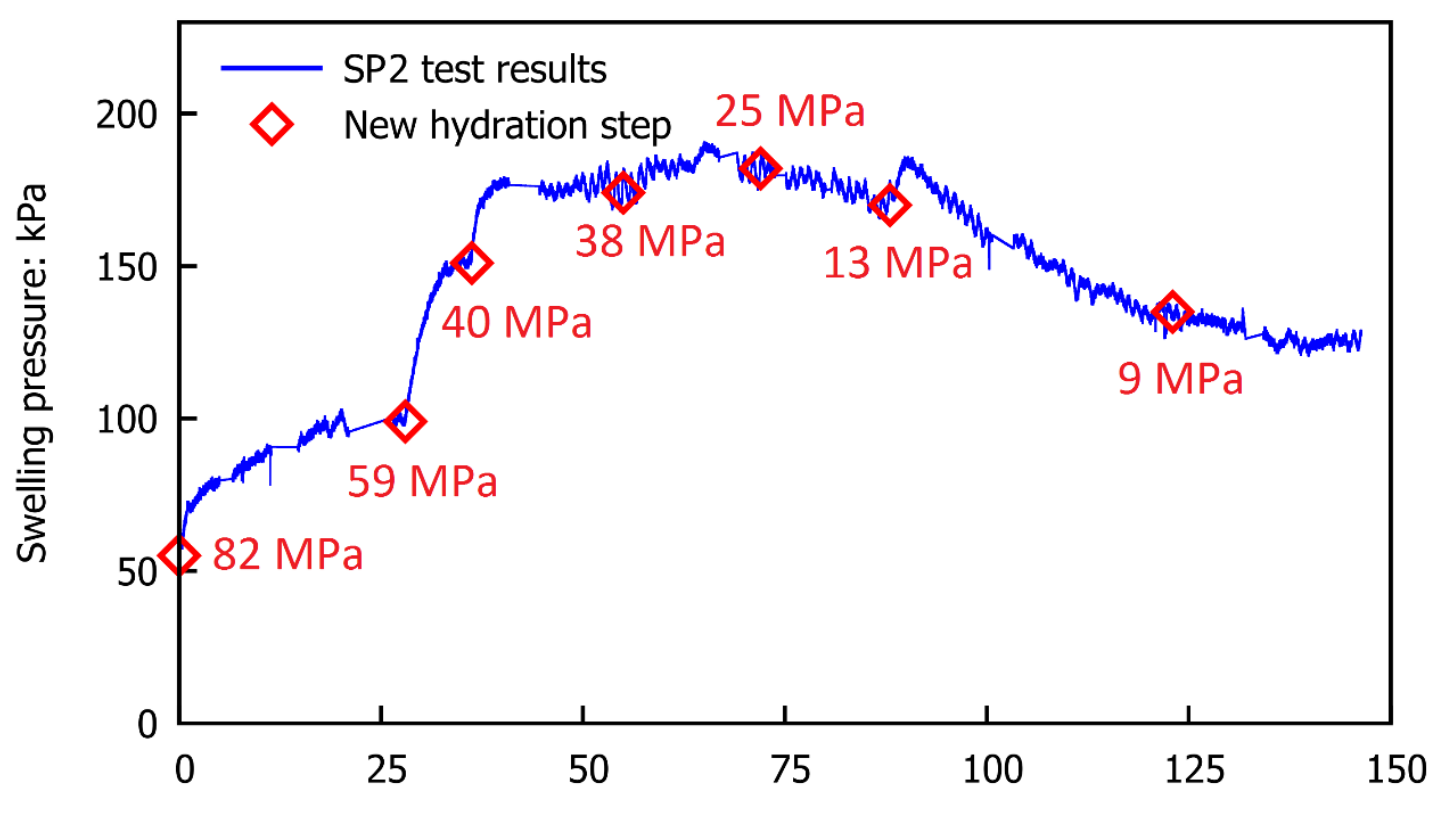

Elapsed time: $d$ 
992 Fig. 9 Swelling pressure as a function of decreasing suction for SP1 and SP2, along with the 993 mean value obtained for DEM1, DEM2 and DEM3 simulations.

994

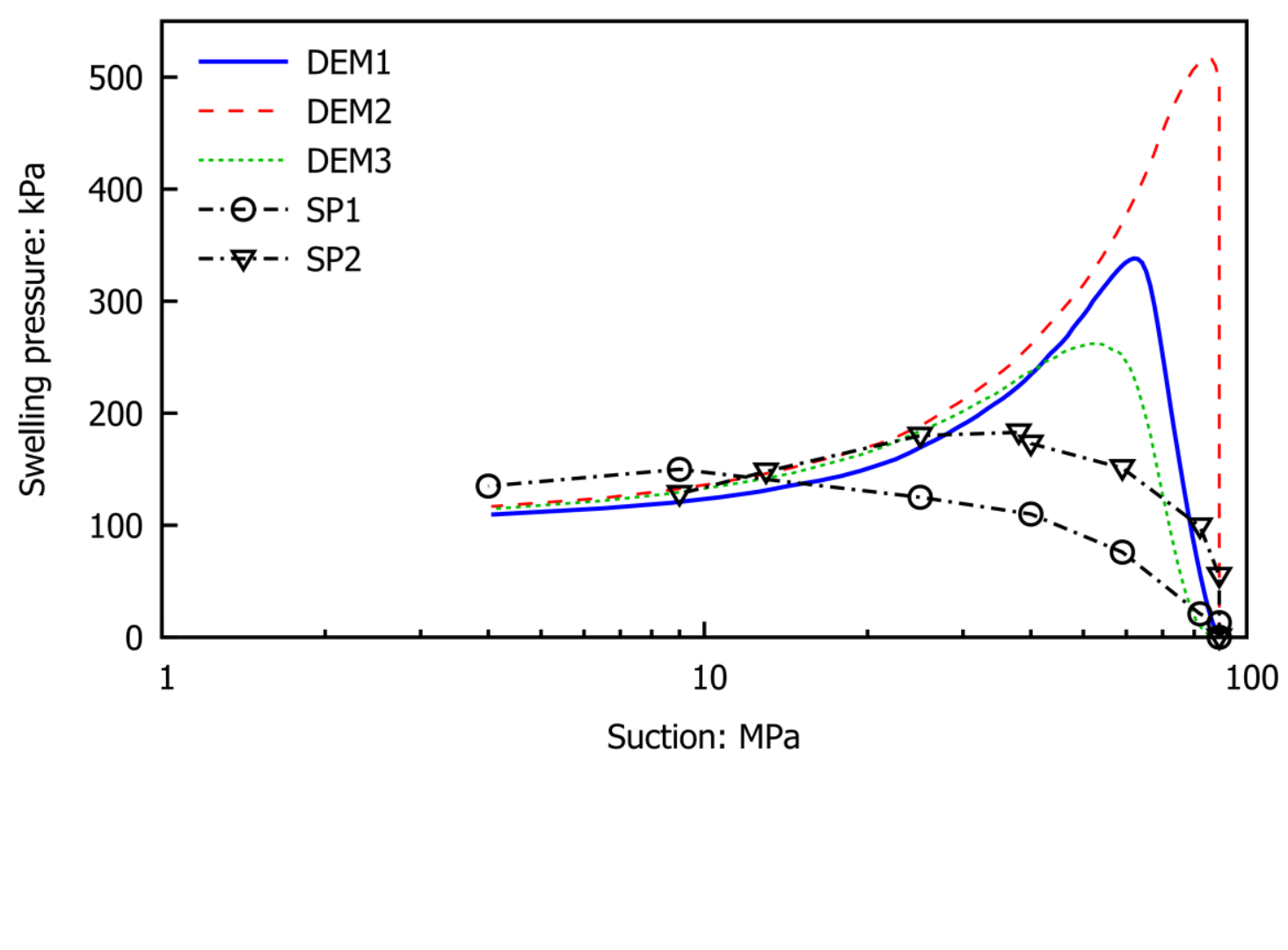


999 Fig. 10a Picture of the SP1 sample after dismantling at $4 \mathrm{MPa}$ of suction. The cell diameter is $60 \mathrm{~mm}$

1001

1002

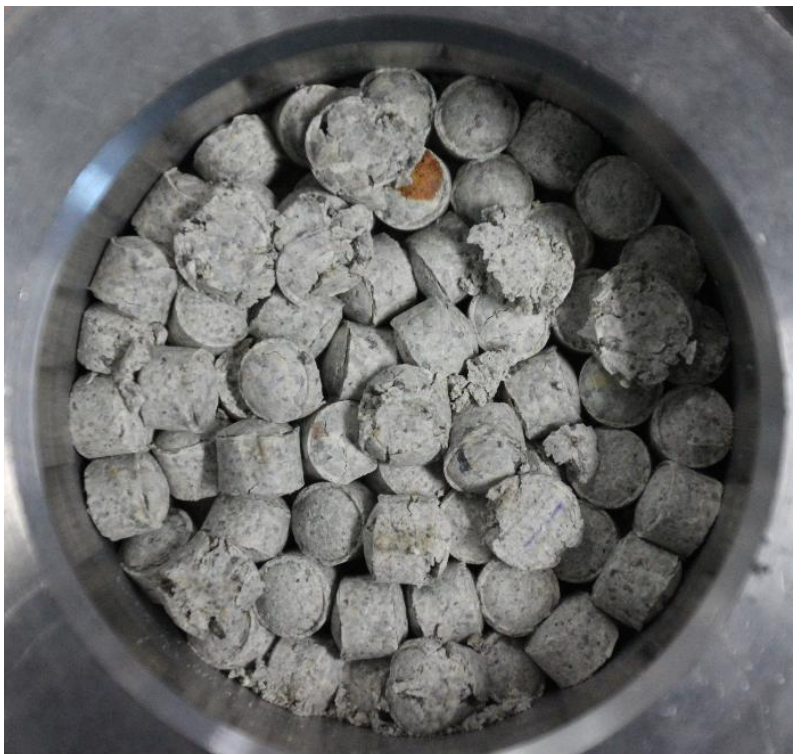

1003

1004

Fig. 10b Picture of the SP2 sample after dismantling at $9 \mathrm{MPa}$ of suction. The cell diameter is $100560 \mathrm{~mm}$

1006

1007

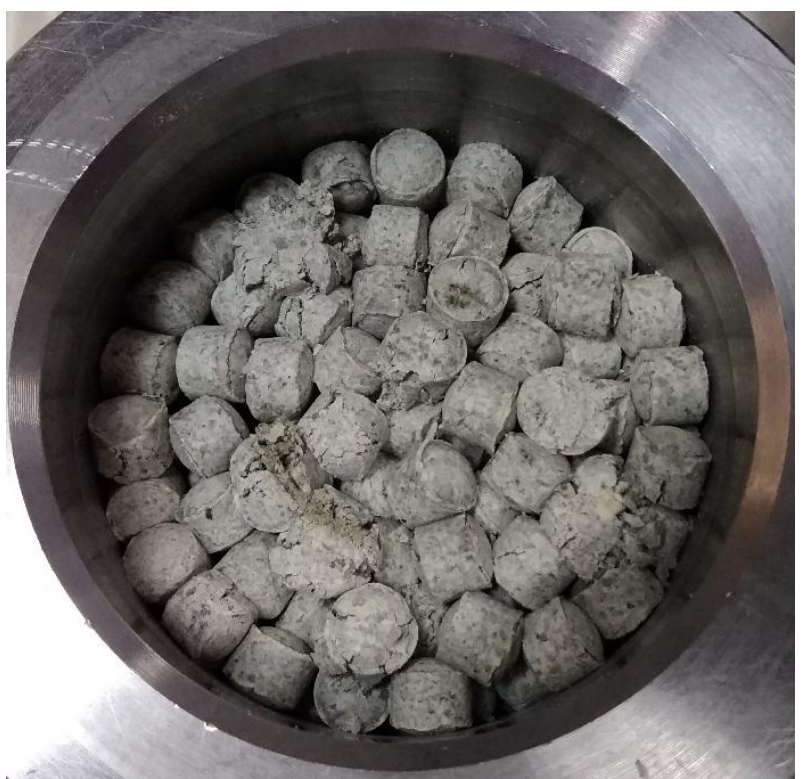

1008

1009

1010 
1011 Fig. 11a Mean value of swelling pressure, proportion of plastic contacts in the sample and 1012 proportion of plastic contacts at the top wall in DEM1

1013

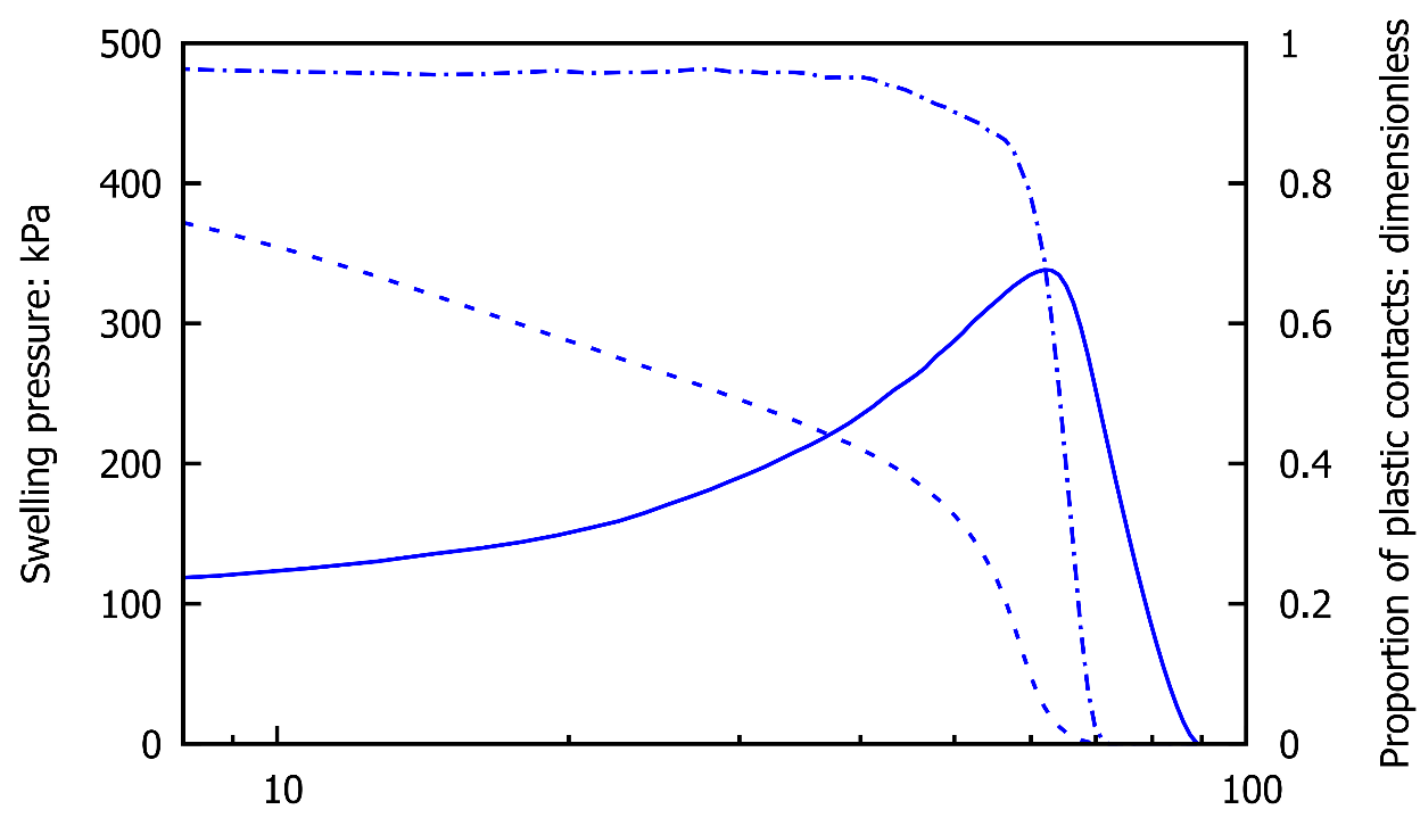

Suction: $\mathrm{MPa}$

1014

DEM simulation results:

Swelling pressure

- - - Plastic contacts (whole sample)

-.-.- Plastic contacts (upper wall)

1015

1016 
1017 Fig. 11b Mean value of swelling pressure, proportion of plastic contacts in the sample and 1018 proportion of plastic contacts at the top wall in DEM2

1019

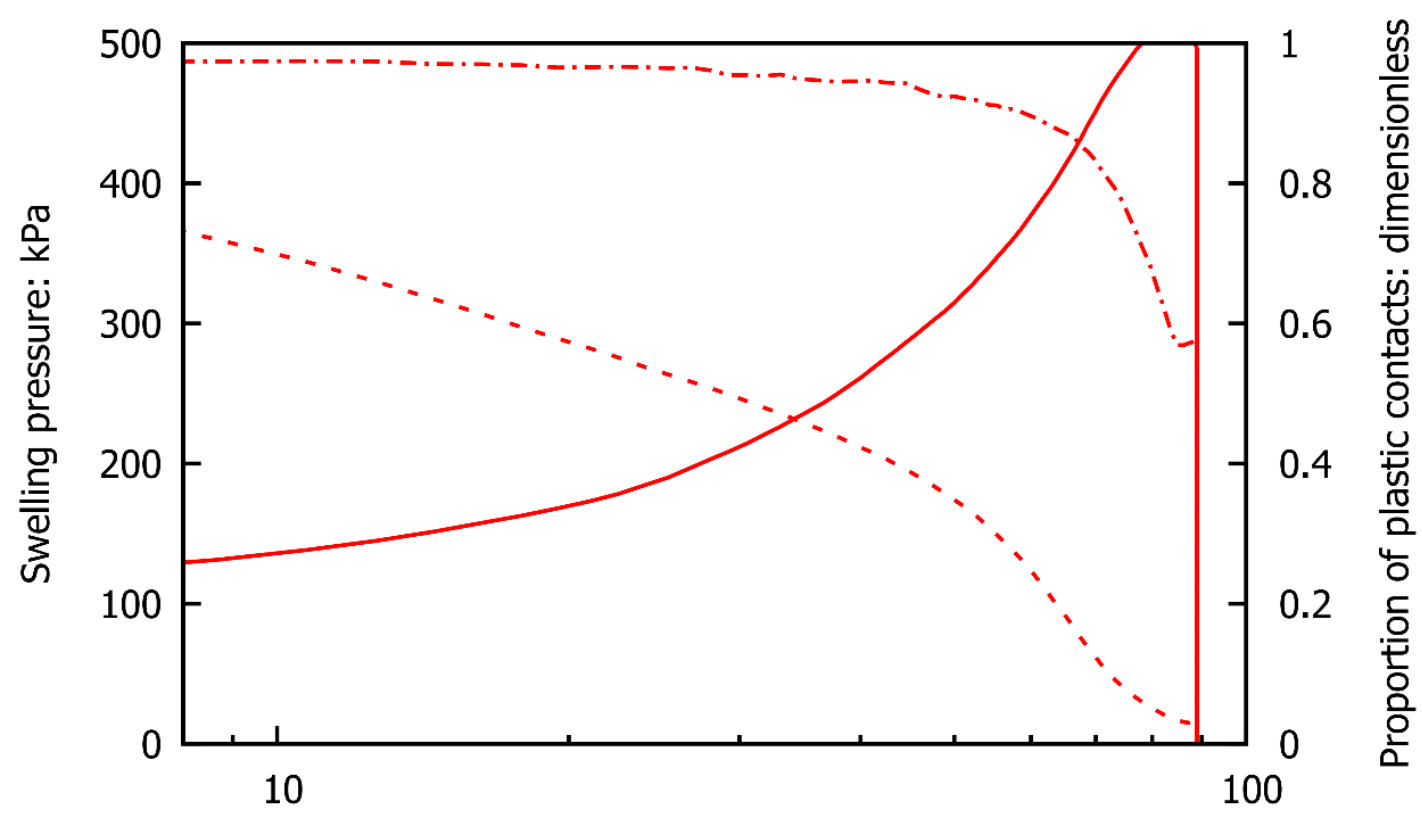

Suction: $\mathrm{MPa}$

DEM simulation results:

Swelling pressure

- - - - Plastic contacts (whole sample)

-.-.- Plastic contacts (upper wall) 
1023 Fig. 11c Mean value of swelling pressure, proportion of plastic contacts in the sample and proportion of plastic contacts at the top wall in DEM3

1025

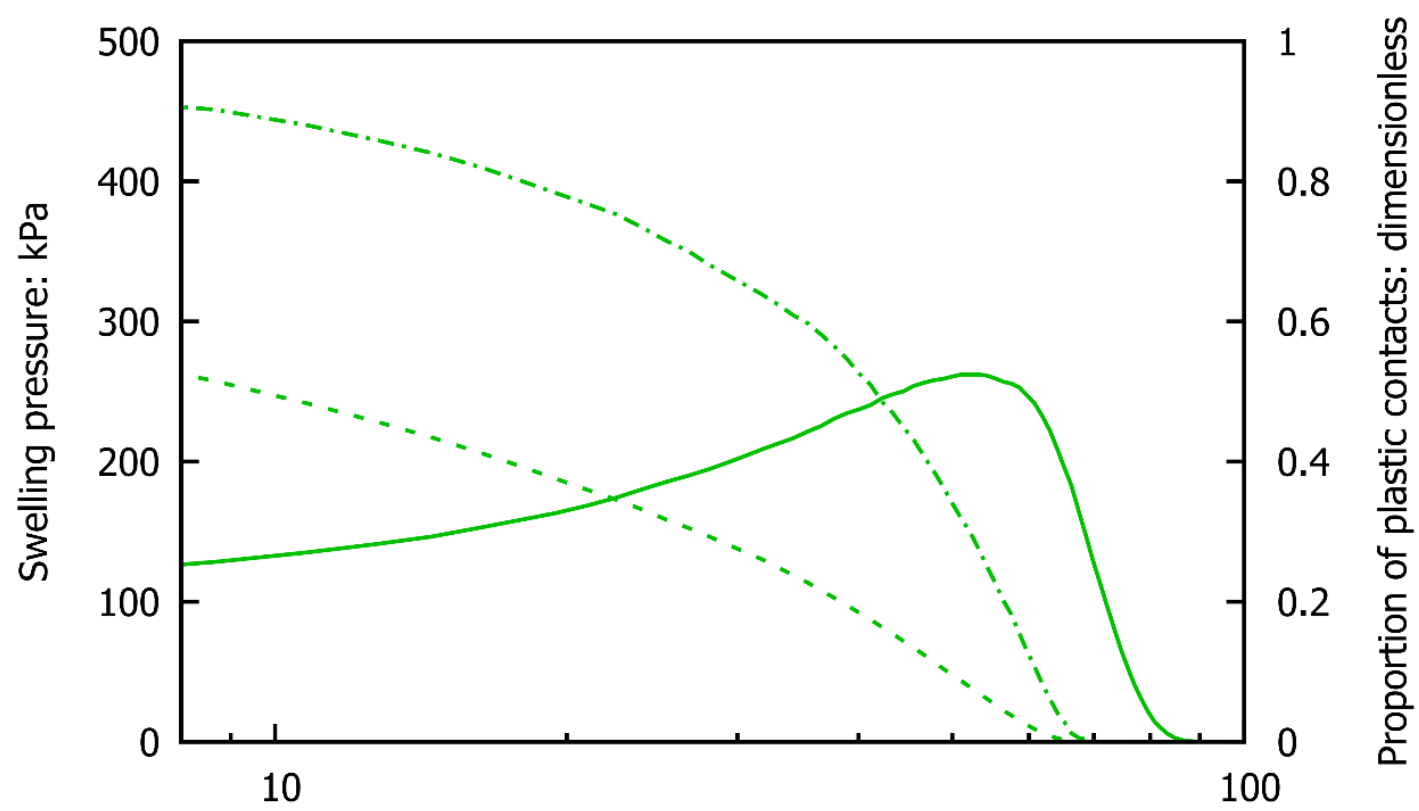

Suction: $\mathrm{MPa}$

DEM simulation results:

Swelling pressure

Plastic contacts (whole sample)

Plastic contacts (upper wall) 
1029 Fig. 12 Coordination number in DEM1, DEM2 and DEM3 simulations (mean values) and evolution of inter-pellet porosity upon suction decrease (identical in all DEM simulations).

1031

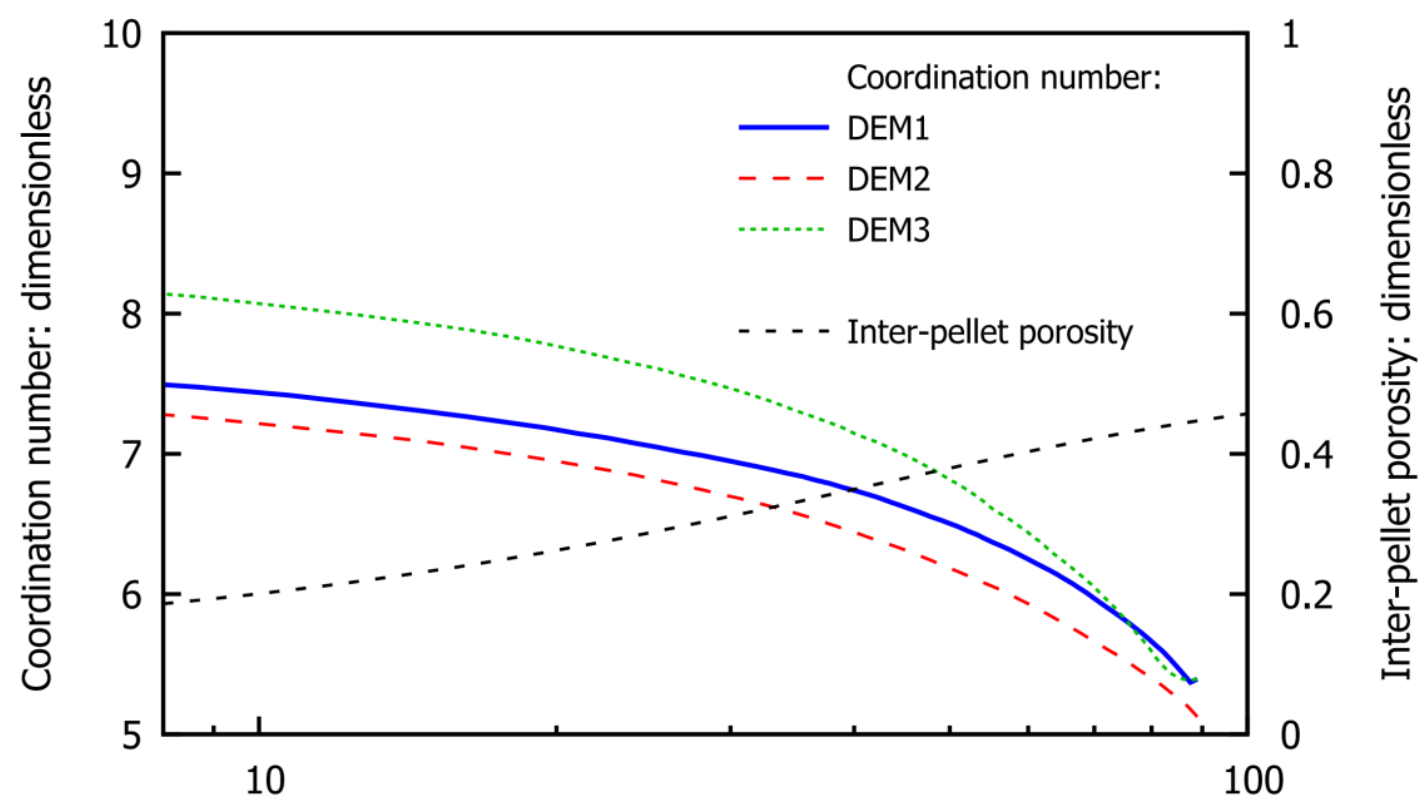

1032

Suction: $\mathrm{MPa}$

1033

1034

1035 
1036 Fig. 13 Coefficient of variation of swelling pressure, proportion of plastic contacts and coordination number in DEM1, DEM2 and DEM3 simulations

1038

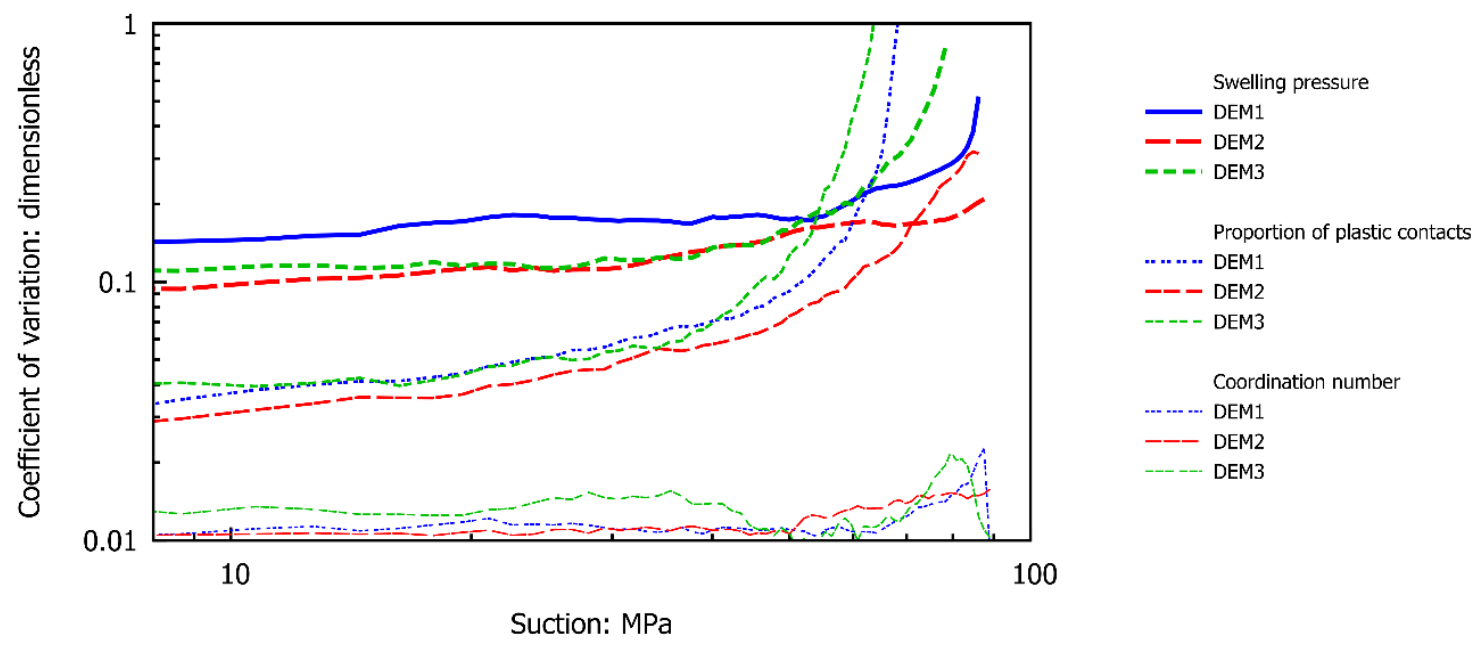

1039

1040

1041 
1042 Fig. 14 Variability of the measured swelling pressure in DEM1 simulations for two sensor sizes 1043

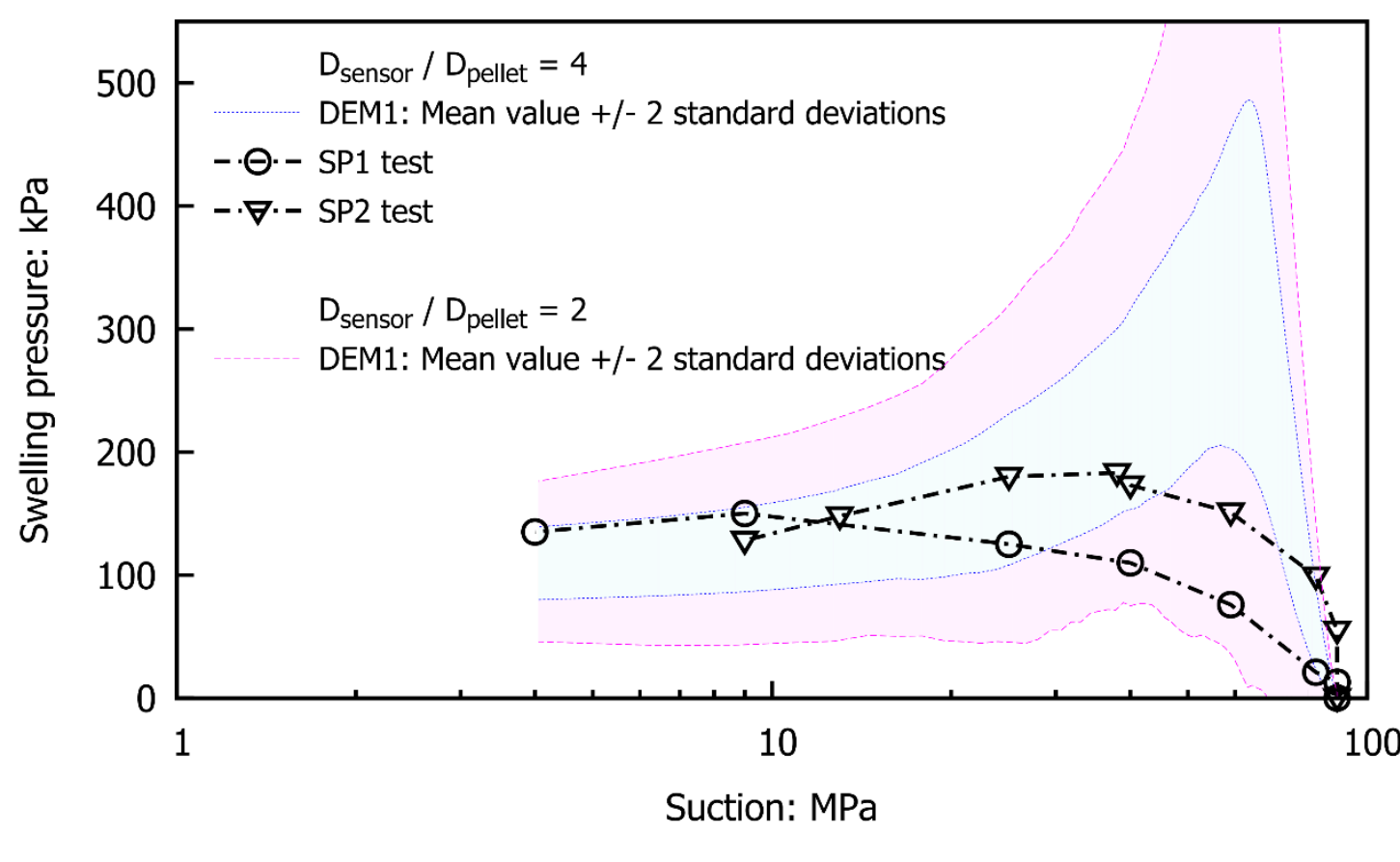

1045

1046

1047 
1048 Fig. 15 Coefficient of variation of the measured swelling pressure in DEM1 simulations at peak 1049 and at $9 \mathrm{MPa}$ of suction

1050

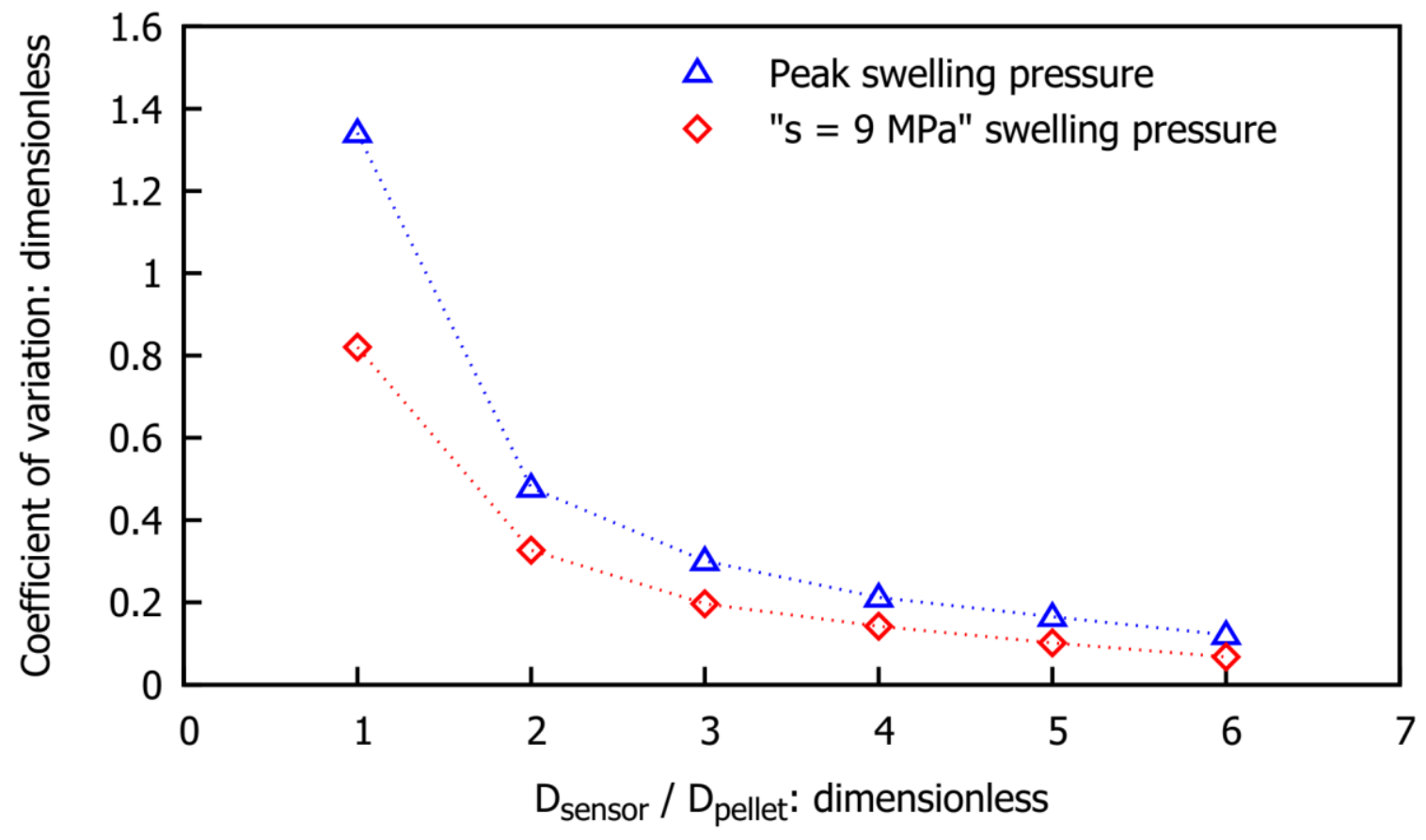

1051

1052

1053

1054 
Fig. 16 Evolution of the mean elastic normal deflection in the numerical sample in DEM1, DEM2 and DEM3 simulations. Mean values are plotted

1057
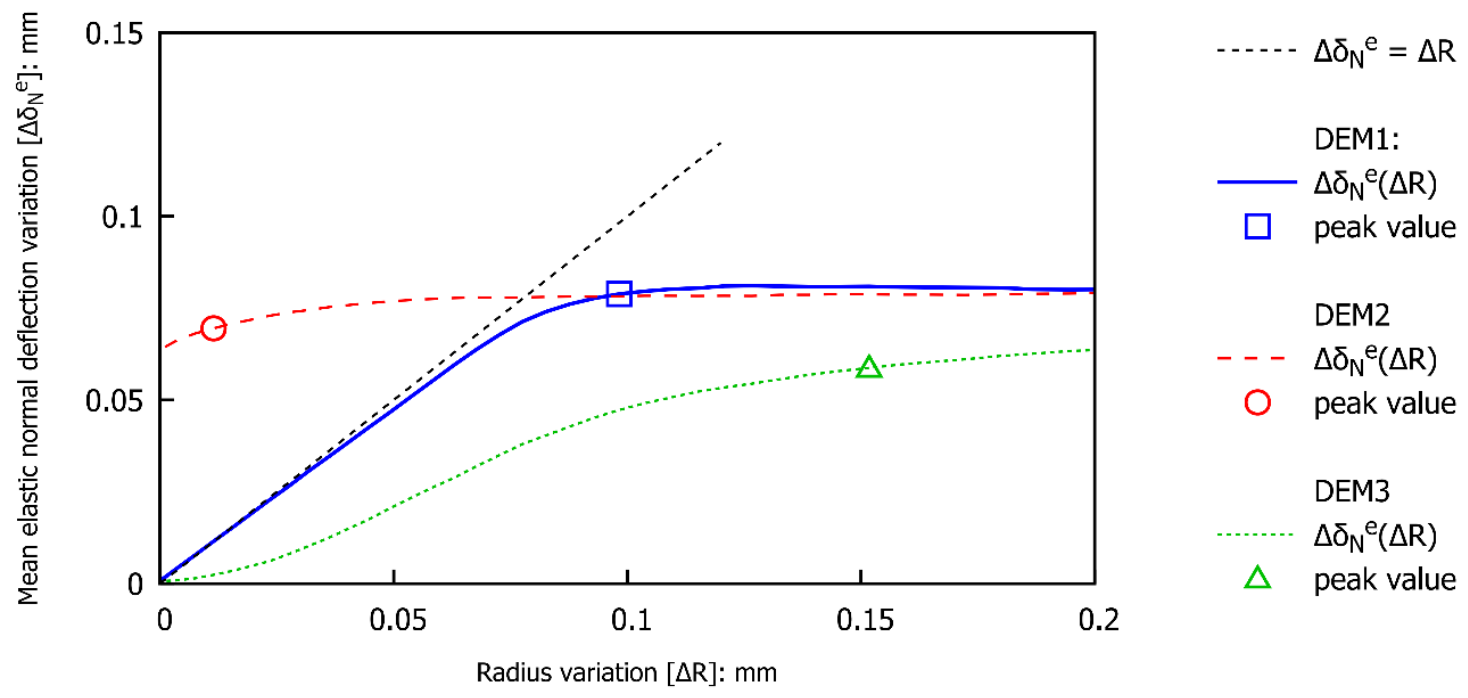

1058

1059

1060 
Fig. 17 Comparison of the mean stress calculated in the sample and the swelling pressure measured by the sensor as functions of decreasing suction for DEM1 simulations

1063

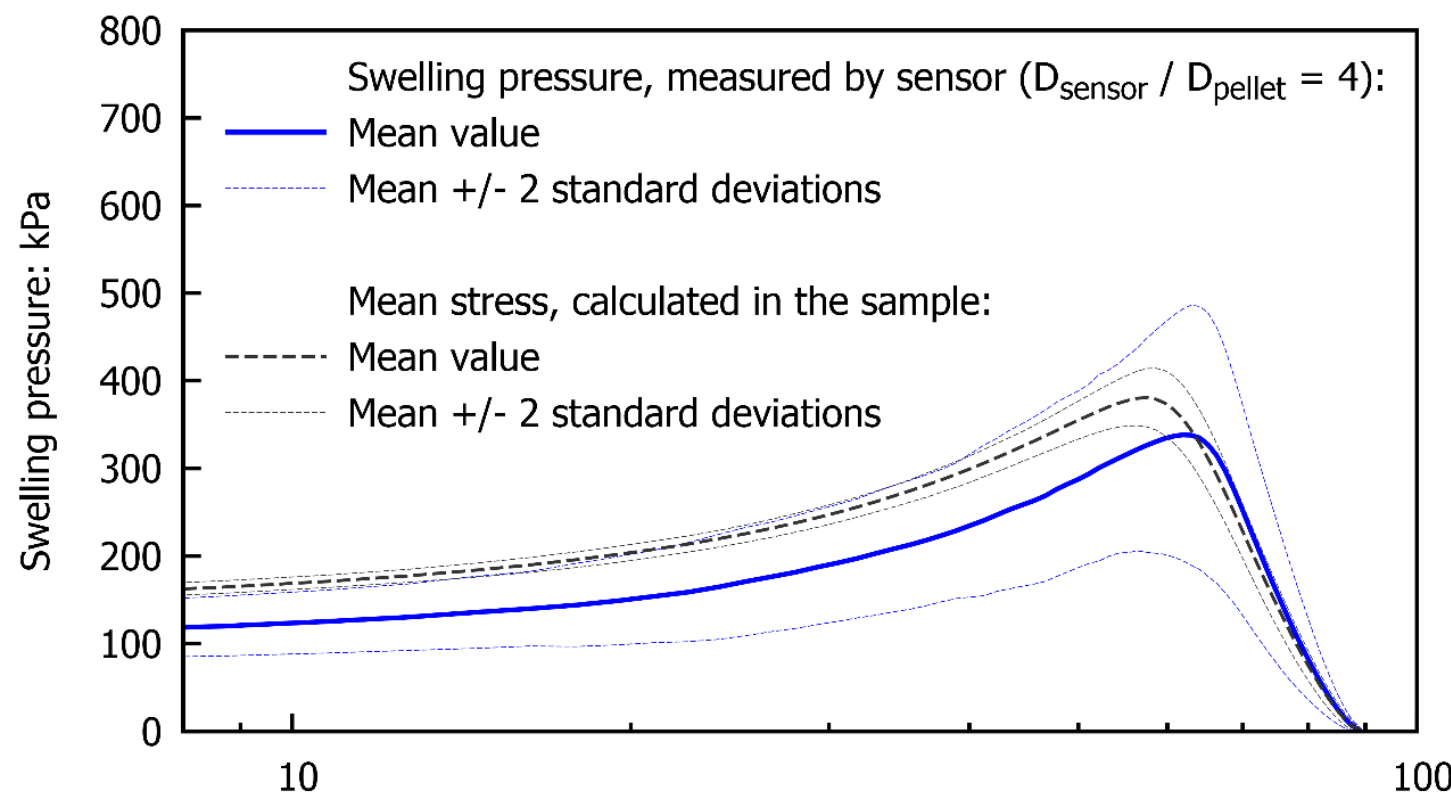

1064

Suction: MPa

1065

1066 